\title{
V1369 Cen High-resolution Panchromatic Late Nebular Spectra in the Context of a Unified Picture for Nova Ejecta
}

\author{
Elena Mason $^{1}$ (D), Steven N. Shore ${ }^{2,3,4}$, Ivan De Gennaro Aquino ${ }^{5}$, Luca Izzo ${ }^{6}$, Kim Page ${ }^{7}$, and Greg J. Schwarz ${ }^{8}$ (D) \\ ${ }^{1}$ INAF-OATS, Via G.B. Tiepolo, 11, I-34143, Trieste, Italy; emason@oats.inaf.it \\ ${ }^{2}$ Dipartimento di Fisica "Enrico Fermi," Universita' di Pisa, Italy \\ ${ }^{3}$ INFN Pisa, Largo B. Pontecorvo, 56127, PI, Italy \\ ${ }^{4}$ Astronomical Institute, Charles University in Prague, V Holešovičkách 2, 180 00, Praha 8, Czech Republic \\ ${ }^{5}$ Hamburger Sternwarte, Gojenbergsweg 112, D-21029 Hamburg, Germany \\ ${ }^{6}$ Instituto de Astrofisica de Andalucia, Glorieta de la Astronomia s/n, E-18008 Granada, Spain \\ ${ }_{7}$ Department of Physics and Astronomy, University of Leicester, Leicester, LE1 7RH, UK \\ ${ }^{8}$ American Astronomical Society, 2000 Florida Ave., NW, Suite 400, DC 20009-1231, USA \\ Received 2017 October 26; revised 2017 December 13; accepted 2017 December 14; published 2018 January 19
}

\begin{abstract}
Nova Cen 2013 (V1369 Cen) is the fourth bright nova observed panchromatically through high-resolution UV +optical multiepoch spectroscopy. It is also the nova with the richest set of spectra (in terms of both data quality and number of epochs) thanks to its exceptional brightness. Here, we use the late nebular spectra taken between day $\sim 250$ and day $\sim 837$ after outburst to derive the physical, geometrical, and kinematical properties of the nova. We compare the results with those determined for the other panchromatic studies in this series: T Pyx, V339 Del (nova Del 2013), and V959 Mon (nova Mon 2012). From this we conclude that in all these novae the ejecta geometry and phenomenology can be consistently explained by clumpy gas expelled during a single, brief ejection episode and in ballistic expansion, and not by a wind. For V1369 Cen the ejecta mass $\left(\sim 1 \times 10^{-4} M \odot\right)$ and filling factor $(0.1 \leqslant f \leqslant 0.2)$ are consistent with those of classical novae but larger (by at least an order of magnitude) than those of T Pyx and the recurrent novae. V1369 Cen has an anomalously high (relative to solar) N/C ratio that is beyond the range currently predicted for a $\mathrm{CO}$ nova, and the Ne emission line strengths are dissimilar to those of typical $\mathrm{ONe}$ or $\mathrm{CO}$ white dwarfs.
\end{abstract}

Key words: stars: novae, cataclysmic variables - stars: individual (V339 Del, V1369 Cen, V959 Mon) - line: formation - line: identification - line: profiles - methods: observational - techniques: spectroscopic

\section{Introduction}

For classical and recurrent novae, the geometry of the ejecta, and its connection to the explosion, the ejection mechanism, the distance and the ejecta mass, have been investigated by several authors. There are various approaches to constraining the geometry. They can be purely geometrical (e.g., PayneGaposchkin 1957; Gill \& O'Brien 1999, 2000; Banerjee et al. 2016), or they can include kinematics (e.g., Chochol et al. 1997; Harman \& O'Brien 2003; Eyres et al. 2005; Munari et al. 2011; Ribeiro et al. 2011, 2013; Linford et al. 2015; Harvey et al. 2016). These studies assume geometrical components to start with, and choose them in combination to fit a model to the observations. In the most sophisticated cases, the best fit indicates components, aspect ratio, maximum velocity, and also mass distribution of the observed ejecta. The dynamics are usually adopted from independent studies, and so is the ejection mechanism.

Attempts to identify the ejection mechanism and solve the long-standing debate on the windy or shell nature of the ejecta include, for example, those of Payne-Gaposchkin \& Menzel (1938), Payne-Gaposchkin (1957), Friedjung (1966a, 1966b), Hutchings (1970), Hjellming (1990), Williams (1992), Scott et al. (1995), Williams et al. (1996), Cassatella et al. (2004b), and Liimets et al. (2012). However, without adequate theoretical modeling of the thermonuclear ignition and the ejecta liftoff to confront with observations, any proposed scenario is potentially equally valid. Since nova ejecta are an expanding medium at relatively high velocity, their physical conditions are constantly changing and out of equilibrium. A successful scenario therefore should explain the observations from the rise phase to the very late decline. In addition, it should be able to make sense of multiwavelength observations across the whole electromagnetic spectrum. With this in mind, we have analyzed and compared multiepoch panchromatic high-resolution spectroscopic observations for a number of recent bright novae that, in view of their brightness, could be followed up to years after outburst.

The use of high-resolution spectroscopy $(R \geqslant 40,000)$ was motivated by the evidence that nova ejecta display clumpy structures. Evidence for clumps has been presented, for example, by early high-resolution photographic spectroscopy (e.g., McLaughlin 1957, 1964; Payne-Gaposchkin 1957), highresolution IUE observations (e.g., Cassatella et al. 2004a, and references therein), and resolved images of old nova remnants (e.g., Gill \& O'Brien 2000; Harman \& O'Brien 2003; Liimets et al. 2012, and references therein; Shara et al. 2015; see also O'Brien \& Bode 2008, and references therein). The effort to obtain panchromatic observations (i.e., contemporaneous, when not simultaneous, UV and optical spectra) was motivated by the need to account for the ionization structure of the ejecta.

Last but not least, because of relatively sparse spectroscopy in the literature of novae in the late nebular stage, we made sure to monitor each of the sufficiently bright novae well after outburst and the supersoft source (SSS) phase. An extended monitoring became compelling after the T Pyx 2011 outburst, which was followed from day 2 to 834 , revealed stationary absorption structures in the UV resonant transitions of the late nebular spectra. Those stationary absorptions, together with the analysis of the pseudo-P-Cyg absorptions during the nova early 
Table 1

Log of Observations for Nova Cen 2013

\begin{tabular}{|c|c|c|c|c|c|c|c|c|c|c|}
\hline \multicolumn{6}{|c|}{ STIS (UV) } & \multicolumn{5}{|c|}{ OPT Instrument } \\
\hline UT Date & $\begin{array}{l}\text { Age } \\
\text { (day) }\end{array}$ & $\begin{array}{c}\text { E140M/ } \\
1425\end{array}$ & $\begin{array}{c}\text { E230M/ } \\
1978\end{array}$ & $\begin{array}{c}\text { E230M/ } \\
2707\end{array}$ & Prop. ID & UT Date & $\begin{array}{l}\text { Age } \\
\text { (day) }\end{array}$ & FEROS & $\begin{array}{c}\text { UVES } \\
\text { DIC1+DIC2 }\end{array}$ & Prop. ID \\
\hline 2014 Aug 12 & 252.7 & $249^{\mathrm{a}}$ & 249 & 249 & 13388 & 2014 Aug 02 & 242.4 & 900 & $\ldots$ & 094.A-9011 \\
\hline 2014 Oct $03^{b}$ & 304.9 & $249^{\mathrm{a}}$ & 249 & 249 & 13388 & & $\ldots$ & $\ldots$ & $\ldots$ & \\
\hline 2015 Mar 06 & 458.4 & 800 & 602 & 601 & 13828 & $\begin{array}{l}2015 \text { Mar } 06 \\
2015 \text { Apr } 06\end{array}$ & $\begin{array}{l}458.5^{\mathrm{c}} \\
489.6\end{array}$ & $\begin{array}{l}\cdots \\
\cdots\end{array}$ & $\begin{array}{r}15 \times 2+35 \times 3+600 \\
100+180+600\end{array}$ & $\begin{array}{l}\text { 095.D-0722 } \\
\text { 095.D-0722 }\end{array}$ \\
\hline 2015 Jun 21 & 566.1 & 800 & 602 & 601 & 13828 & 2015 Jun 21 & 566.3 & $\ldots$ & $100+180+600$ & 095.D-0722 \\
\hline 2016 Mar 19 & 837.7 & 2679 & 1440 & 1439 & 14338 & 2016 Mar 17 & 835.5 & $\cdots$ & $100 \times 2+200+900$ & 096.D-0226 \\
\hline
\end{tabular}

Notes. For each instrument/instrument setup the total integration time is given in seconds. The nova age is given in days since outburst/discovery $\left(T_{0}\right.$ is 2013 December 2.692UT or MJD $=56,628.69$ ).

${ }^{a}$ In this case the FUV setup was grism E140H centered at 1234, 1416, and $1598 \AA$. Each setup had exposure $249 \mathrm{~s}$. The spectral resolution of the E140H is $R=114,000$.

${ }^{\mathrm{b}}$ The object was not visible from ground at the time of the HST observations.

${ }^{c}$ Low-S/N data therefore are not considered for quantitative measurements in the text/paper.

decline, showed that the ejecta are in ballistic expansion and consistent with a biconical geometry (Shore et al. 2011, 2013b; De Gennaro Aquino et al. 2014). Hence, panchromatic Target of Opportunity (ToO) and long-term monitoring campaigns were organized to follow any bright nova through highresolution UV+optical spectroscopy. Complementary Swift observations and photometric monitoring (AAVSO; Kafka 2017) were used to program the cadence and the exposure time of the observations. The bright novae that could be followed by our programs were V339 Del (nova Del 2013), V1369 Cen (nova Cen 2013), and in part V959 Mon (nova Mon 2012).

Partial results have already been published for V959 Mon (Shore et al. 2013a) and V339 Del (Shore et al. 2016) showing that the biconical geometry can describe those two objects as well. In addition, early spectra of V339 Del (V959 Mon was discovered during its nebular phase) displayed absorption structures whose development was quite similar to those of $\mathrm{T}$ Pyx (Shore et al. 2011).

This paper presents an analysis of the late nebular spectra of V1369 Cen and compares the results with the late nebular spectra collected for the panchromatic novae listed above and not yet published. The major conclusion of this paper goes beyond the physical parameters derived for the ejecta of V1369 Cen and underlines the commonalities among the individual spectroscopic evolutions.

Every individual object for which we have collected comparable data sets is essentially different in its physical parameters, development timescale, and detailed line profiles. However, from their comparative analysis it emerges that a single assumption (the single explosion) allows us to describe each phenomenon within a single unified scenario: the visible (at any time and any wavelength) nova ejecta are not a wind, but are rather consistent with an ensemble of structures in ballistic or free expansion ( $v \sim r$, also called a Hubble flow) confined within a biconical geometry.

The paper consists of several parts. The first part presents the data collection and selection (Sections 2 and 3). The second part uses the data to characterize V1369 Cen quantitatively, describing its spectral evolution and deriving all the possible physical parameters of the nova (Sections 4 and 5). Section 6 analyzes transitions and line profiles to constrain the ejecta ionization structure and geometry. The line profile analysis is exemplified using V1369 Cen spectra, but the results are presented together with those derived for T Pyx, V339 Del, and V959 Mon in the previous publications. We discuss the implications of our results in Section 7. We will show in particular that our proposed scenario is consistent not only with each of the collected high-resolution panchromatic epochs (from day 0 to, virtually, the undetectability) but also with recent results from other regimes of the electromagnetic spectrum. Summary and conclusions are in Section 8.

\section{Observations and Data Reduction}

\subsection{Panchromatic High-resolution Spectroscopy}

For each object, the panchromatic data set consists of coordinated optical and UV spectra. Early epochs had the requirement of nearly simultaneous observations, while the late nebular spectra presented herein could be up to 1 month apart for easy scheduling. UV spectra were secured with the Space Telescope Imaging Spectrograph (STIS) on board the Hubble Space Telescope (HST). The adopted instrument setup was generally the same using the medium-resolution echelle gratings E140M centered at $1425 \AA$ and E230M centered at 1978 and $2707 \AA$ on consecutive exposures. The resulting UV range was $1200-2900 \AA$ with spectral resolution $R=45,800$ and $R=30,000$ for the far-UV (FUV) and near-UV (NUV) band, respectively. The exception was early in the V1369 Cen outburst when the nova was still too bright to be safely observed with the medium echelle in the FUV. Then, the highresolution echelle was used; see Table 1.

The optical spectra were obtained either at the Nordic Optical Telescope (NOT) using the FIbre-fed Echelle Spectrograph (FIES) for the case of the northern objects (e.g., V339 Del) or with the Fiber-fed Extended Range Optical Spectrograph (FEROS) at the $2.2 \mathrm{~m}$ telescope and the Ultraviolet and Visual Echelle Spectrograph (UVES) at the Very Large Telescope (VLT) for the southern objects (V1369 Cen). The covered optical range varied slightly depending on the instrument, the largest being offered by UVES, which is the only one capable of covering the whole visible band from the atmosphere cutoff at 3000-10000 $\AA$. We took advantage of this capability by combining the dichroic setups 
Table 2

Log of Observations for Nova Del 2013

\begin{tabular}{|c|c|c|c|c|c|c|c|c|c|}
\hline \multicolumn{6}{|c|}{ STIS (UV) } & \multicolumn{4}{|c|}{ OPT Instrument } \\
\hline UT Date & $\begin{array}{l}\text { Age } \\
\text { (day) }\end{array}$ & E140M/1425 & E230M/1978 & E230M/2707 & Prop. ID & UT Date & $\begin{array}{l}\text { Age } \\
\text { (day) }\end{array}$ & FIES & Prop. ID \\
\hline 2015 May 11 & 635.3 & 2455 & 1328 & 1328 & 13828 & 2015 May 27 & 651 & 2000 & P51-103 \\
\hline 2015 Sep $19^{a}$ & 765.5 & 2455 & $\ldots$ & $\ldots$ & 13828 & $\ldots$ & $\ldots$ & $\ldots$ & $\ldots$ \\
\hline 2015 Dec 29 & 866.4 & 2455 & 1238 & 1237 & 13828 & $\ldots$ & $\ldots$ & $\ldots$ & $\ldots$ \\
\hline
\end{tabular}

Note. For each instrument/instrument setup the total integration time is given in seconds. The nova age is given in days since outburst/discovery ( $T_{0}$ is 2013 August 14.58UT or MJD $=56,518.58$ ).

${ }^{\text {a }}$ The 2015 September 19 HST visit failed (only the FUV setup could be obtained) and was repeated on 2015 December 29 . At both epochs the optical spectrum could not be obtained.

Table 3

Log of Observations for Nova Mon 2012

\begin{tabular}{|c|c|c|c|c|c|c|c|c|c|c|}
\hline \multicolumn{6}{|c|}{ STIS (UV) } & \multicolumn{5}{|c|}{ OPT Instrument } \\
\hline \multirow{2}{*}{ UT Date } & \multirow{2}{*}{$\begin{array}{l}\text { Age } \\
\text { (day) }\end{array}$} & \multirow{2}{*}{ E140M/1425 } & \multirow{2}{*}{ E230M/1978 } & \multirow[t]{2}{*}{$\begin{array}{c}\text { E230M/ } \\
2707\end{array}$} & \multirow{2}{*}{ Prop. ID } & \multirow{2}{*}{ UT Date } & \multirow{2}{*}{$\begin{array}{l}\text { Age } \\
\text { (day) }\end{array}$} & \multicolumn{2}{|l|}{ UVES } & \multirow{2}{*}{ Prop. ID } \\
\hline & & & & & & & & DIC1 & $\overline{\mathrm{DIC} 2}$ & \\
\hline $\begin{array}{c}2015 \mathrm{Mar} \\
3 / 4 / 7\end{array}$ & $988^{a}$ & $2441 \times 2+2970 \times 2$ & $2411+2970$ & 2970 & 13828 & 2015 Feb 18 & $974^{\mathrm{a}}$ & $1100 \times 2+2160$ & 2160 & 095.D-0722 \\
\hline
\end{tabular}

Note. For each instrument/instrument setup the total integration time is given in seconds.

${ }^{a}$ For nova Mon 2012, the age is counted from the first $\gamma$-ray detection (2012 June 19; Fermi Collaboration 2014).

DIC $1 / 3460+5800 \AA$ and DIC $2 / 4370+8600 \AA$. FIES and FEROS have fixed spectral format and cover, respectively, the wavelength range 4000-7500 $\AA$ and 4000-9000 $\mathrm{A}$. All spectra had high resolution: $R=48,000$ for FEROS, $R=45,000$ or 67,000 for FIES, and $R=40,000$ in UVES.

The detailed log of observations is provided in Tables 1-3. Note that, while performed, the V959 Mon STIS observations failed, so no UV spectrum exists for the last epoch of observation of V959 Mon (day 988).

STIS data were reduced with the instrument pipeline. The order merging was, however, performed with the HST Goddard High Resolution Spectrograph script because of its better performance. Instrument pipeline reductions were also used for the optical spectra. We flux-calibrated FEROS and FIES data using our own scripts (IDL and/or IRAF). For each object we observed a standard star that was relatively close in pointing direction. The standard-star spectra (BD $+28^{\circ} 4211$ for V339 Del at FIES and LTT $4816+\mathrm{CD}-32^{\circ} 9927$ for V1369 Cen at FEROS) were taken immediately after the science observation. Although we also observed dedicated standard stars for the UVES runs (HR 2315 for V959 Mon and LTT 4816 for V1369 Cen), we finally adopted the master response function delivered by ESO for its better response below $3500 \AA$ and above $9000 \AA$. We ascertained that the master response function matched the response function built with the dedicated standard star in the overlap range.

\subsection{The X-Ray Light Curve}

As mentioned in Sections 1 and 3, the nova X-ray light curve was used to aid the planning of the observations and the identification of the nebular spectra. The V1369 Cen light curve obtained from Swift observations is shown in Figure 1, together with the hardness ratio (HR) evolution.

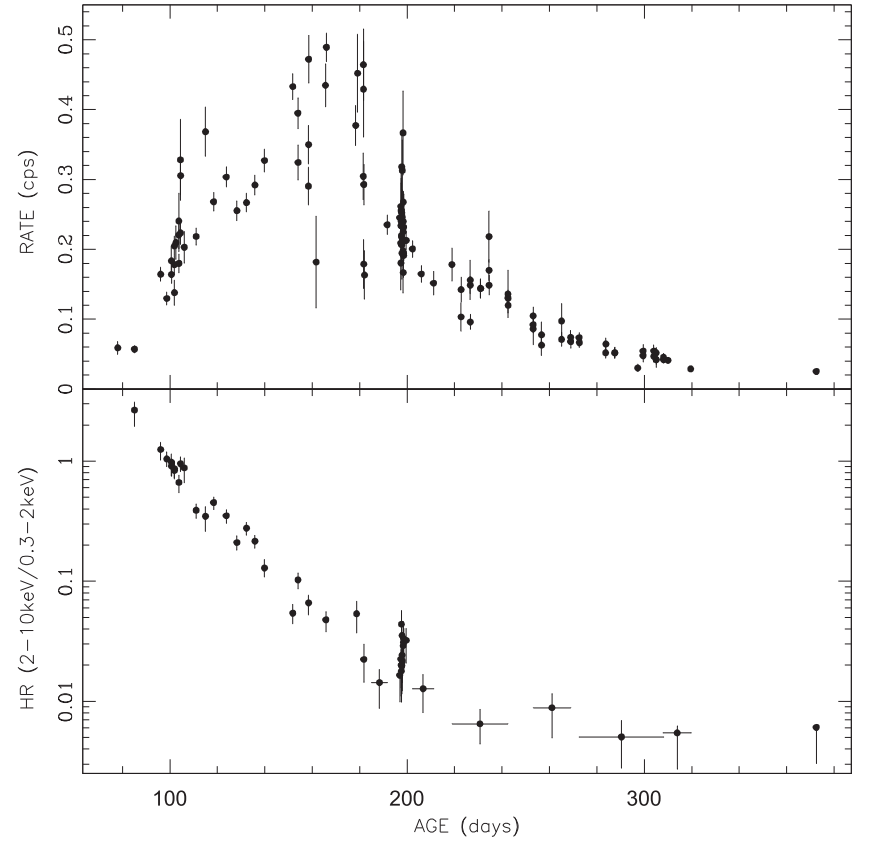

Figure 1. V1369 Cen X-ray (top panel) and HR (bottom panel) light curves obtained from Swift-XRT observations.

The Swift-XRT data were processed and analyzed using the standard HEASoft tools. The optical brightness of the nova was such that the pileup of optical photons up to X-ray energies ("optical loading") was a concern. Thus, the light curve was obtained extracting only single-pixel (grade 0) events, which helps to mitigate this problem. In addition, the earliest data (up until 2014 February 18; day 78) were obtained using Windowed Timing (WT) mode, which further decreases the optical loading issues, due to the faster read-out time. 
At no point was there any sign of X-ray pileup in the data, so the source light curve and HR bins were extracted using circular regions with radii of $10-20$ pixels $(1$ pixel $=2$ !" 36) depending on the brightness of the source. For the WT data, the background determination was performed using the same size region, offset from, but close to, the source; for Photon Counting mode, a larger circular region was used.

\section{Selection of the Late Nebular Spectra Data Sets}

The reason for concentrating on the late nebular spectra is that they provide essential information about the ejecta structure, kinematics, physics, and geometry as we will show. The late nebular spectra are also somewhat easier to deal with than the spectra at earlier stages because they are optically thin. These spectra, however, need to be carefully identified. A nova spectrum has usually been called "nebular" as soon as [O III] $\lambda \lambda 5007,4958$ appears. Observationally, this occurs at the beginning of the SSS phase, i.e., when the ejecta are gradually turning optically thin but the density is still high enough that collisions are an important cooling mechanism. Collision and photoionization both affect the ejecta substructures, depending on their positions and densities.

Here we define as "late nebular" the data collected once the ionization structure of the ejecta has frozen and the ejecta cooling is driven by recombination alone, which is mediated by the expansion of the gas (e.g., Vanlandingham et al. 2001). This stage can be identified a priori from the inspection of the $\mathrm{X}$-ray light curves (total count and HR) and verified a posteriori from the inspection of the line profiles (which no longer change) and the development of the integrated line fluxes (which decrease as a power law). For each nova we set the start of the late nebular phase on the basis of the visual inspection of the X-ray and HR light curves. In this way, late nebular spectra are those obtained after day 250 for the case of V1369 Cen, when the count rate dropped below $0.10 \mathrm{cps}$ and the HR was less than 0.01 (see Figure 1); after day 280 for V339 Del, when the count rate had dropped by almost four orders of magnitude with respect to maximum to $\sim 0.01 \mathrm{cps}$ and the HR was of the order of 0.1 (see Figure 1 of Shore et al. 2016); and after day 250 for V959 Mon, when the count rate was about one order of magnitude below maximum at $\sim 0.1$ counts $s^{-1}$ (see Figure 1 of Page et al. 2013).

\section{V1369 Cen Spectral Characteristics and Evolution}

In this and the following section we place V1369 Cen in context determining qualitative and quantitative information similar to that in our studies of T Pyx, V339 Del, and V959 Mon (T Pyx, Shore et al. 2011, 2013b; De Gennaro Aquino et al. 2014; V339 Del, Shore et al. 2016; V959 Mon, Shore et al. 2013a). V1369 Cen offers the most valuable sequence for the study of line profile evolution after the turnoff of the SSS, since we have (see Table 1) five epochs between day 252 and 837 and exquisite signal-to-noise ratio $(\mathrm{S} / \mathrm{N} ;>10$ and $\geqslant 50$ in the UV and optical continuum, respectively, in the last-epoch spectra). We note that day 252 was at least $\sim 50$ days after $\mathrm{X}$-ray maximum.

On day 252/242 (where the two numbers indicate the nova age at the time of the UV and optical spectroscopy, respectively) the observed emission lines are those of a highly ionized gas enriched in CNO. The gas emission measure is still fairly large as shown by the line width (FWHM $\sim 1300 \mathrm{~km} \mathrm{~s}^{-1}$ ) and the extended wings (up to $\pm 1300 \mathrm{~km} \mathrm{~s}^{-1}$ from the line center). The line profiles are either "flat-top" or "saddle" shaped (see the Appendix). The resonant transitions ( $\mathrm{N} \mathrm{V} \lambda \lambda 1238,1242$, C IV $\lambda \lambda 1548,1550$, and $\mathrm{C}$ II $\lambda \lambda 1334,1335)$ also show a weak and relatively narrow absorption trough in the range $\sim-1000$ to $-1700 \mathrm{~km} \mathrm{~s}^{-1}$.

The STIS spectrum of day 304 is nearly identical to that of day 252: it displays almost the same continuum level (with just a slight change in slope, being steeper in the later epoch) and a small decrease in the line flux.

A significant change in the line profile is observed from the day 458/489 spectrum (see the Appendix), especially in the UV range. The spectra from day $458 / 489$ to day $837 / 835$ show the same dominant species and line profiles and differ in the line fluxes because of the reduced emission measures. The emission component is confined to narrow peaks at velocity $-600 \mathrm{~km} \mathrm{~s}^{-1}$ and $+500 \mathrm{~km} \mathrm{~s}^{-1}$, and the resonance lines that showed narrow absorption troughs now display multiple discrete absorptions in the range $\sim-700$ to $-2000 \mathrm{~km} \mathrm{~s}^{-1}$. Forbidden and recombination transitions display similar emission peaks, but they also show substantial emission at low velocities in the range $-600 \mathrm{~km} \mathrm{~s}^{-1}<v_{\mathrm{rad}}<+500 \mathrm{~km} \mathrm{~s}^{-1}$. The peaks appear more pronounced in the higher excitation transitions and in the $[\mathrm{Ne}$ III $] \lambda \lambda 3869,3968$ and [N II] $\lambda \lambda 5755,6583,6548$ lines. The weak UV lines have decreased in flux, so the spectrum is now dominated by N v $\lambda \lambda 1548,1550$, C II $\lambda \lambda 1334,1335$, O IV $\lambda \lambda 1401 ， 1405$, N IV] $\lambda \lambda 1483,1486, C$ IV $\lambda \lambda 1548,1550$, He II $\lambda 1640$, and C III] $\lambda \lambda 1906$, 1908. In the optical, thanks to the larger wavelength coverage of the UVES spectrum, we detect O III lines pumped by the Bowen fluorescence mechanism (e.g., $\lambda \lambda 3132,3340,3444$, and 3759) and [Ne v] $\lambda \lambda 3346,3426$. The optical lines have also weakened, but emission from [Ar V] $\lambda 7006$ and [Fe VII] (e.g., $\lambda \lambda 3586,5158$, and 5721) is now relatively stronger.

One aspect worth stressing for V1369 Cen is the observed set of neon lines and their relative strengths compared with the other nova spectra. V959 Mon was classified as an ONe nova on the basis of its comparison (and very close match) with the nova Vel 1999, nova Cyg 1992, and nova LMC 2000 spectra at similar ages (Shore et al. 2013a), and V339 Del was classified as a CO nova by comparison with OS And (Shore et al. 2016). V1369 Cen does not resemble any of those objects. Its Ne line strengths are atypical for an ONe nova, since it never develops strong UV Ne transitions, while [Ne III] and [N V] lines are quite strong, in particular, much stronger than in $\mathrm{CO}$ novae at any stage (see Figure 2). Moderate $\mathrm{Ne}$ enrichment is expected in $\mathrm{CO}$ novae since during the He-burning phase ${ }^{14} \mathrm{~N}$ is converted into ${ }^{22} \mathrm{Ne}$, or in case the nova experienced the CNO breakout during the thermonuclear runaway (TNR; e.g., Livio \& Truran 1994; José \& Shore 2008). V1369 Cen is likely one of the moderately Ne-enriched nova as defined by Livio \& Truran (1994).

\section{V1369 Cen Physical Parameters}

\subsection{Reddening}

From a series of 12 spectra (FEROS, $R=48,000)$ taken during early decline, we measured an equivalent width $\mathrm{EW}=0.345 \pm 0.005 \AA$ for the Na I D2 $\lambda 5890$ interstellar 

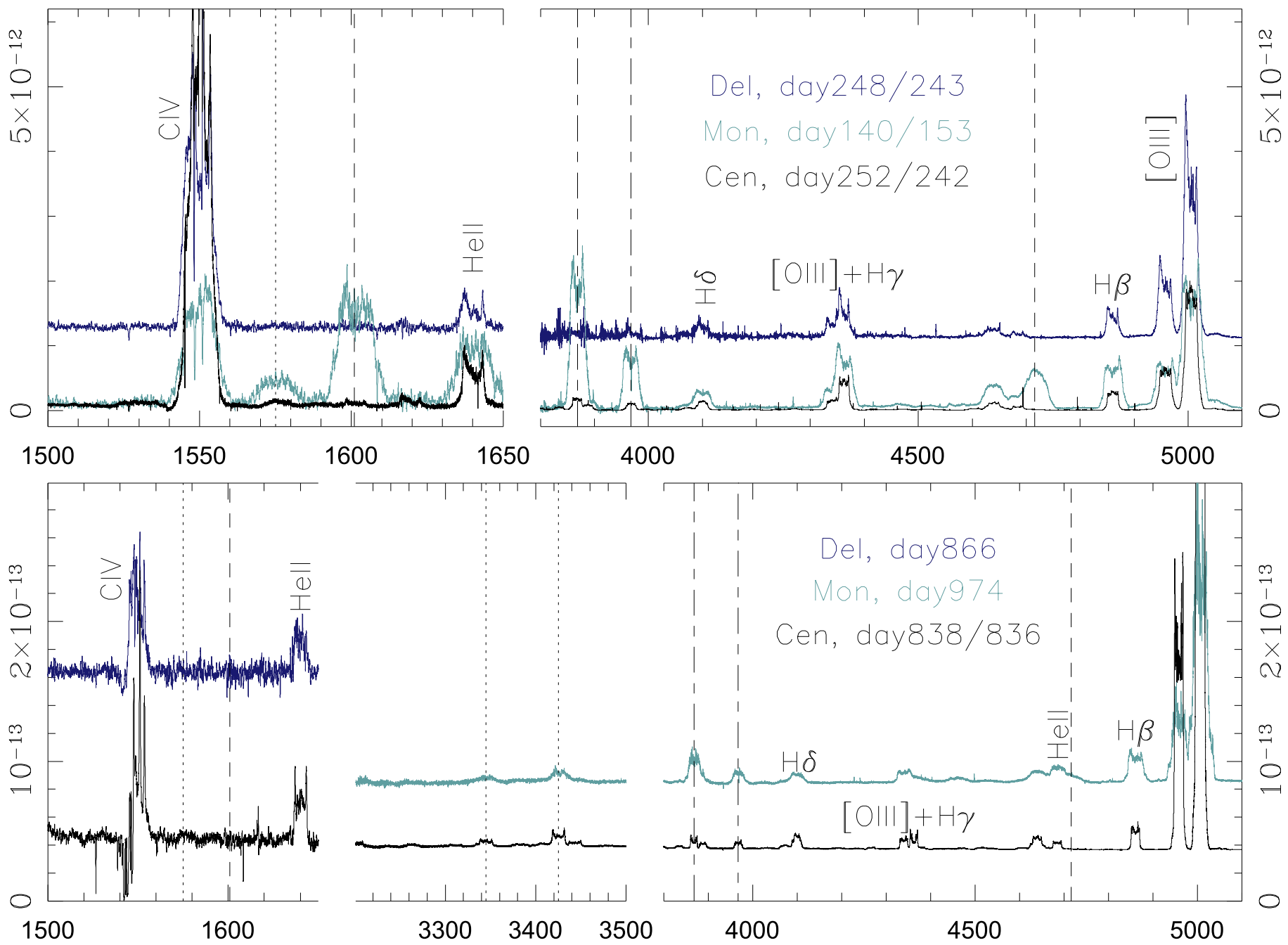

Figure 2. Comparison of V1369 Cen, V339 Del, and V959 Mon Ne emission lines at distinct epochs. Epochs and color-code identification of each spectrum are in the figure itself. The abscissa is in $\AA$, and the ordinate is in $\mathrm{erg} \mathrm{s}^{-1} \mathrm{~cm}^{-2} \AA^{-1}$. Note that the top and bottom panels cover different wavelength ranges. All spectra, except V1369 Cen optical spectra of days 242 and 835 , have been smoothed with a running boxcar filter of size 5-19 points, depending on the S/N of the spectrum. The spectra have been scaled and shifted for an easier comparison: in the top panel, V339 Del has been shifted by the constant $1.1 \times 10^{-12}$, V959 Mon has been scaled by a factor of 10, and V1369 Cen has been scaled by a factor of 1/18; in the bottom panel, V339 Del has been shifted by the constant $1.5 \times 10^{-13}$, V339 Del has been scaled by a factor of 20 and shifted by the constant $0.8 \times 10^{-13}$, and V1369 Cen has been scaled by a factor of 0.2 . No spectrum has been corrected for reddening. The vertical lines mark the Ne transitions: the dotted line is for [Ne V] $\lambda 1575$ and $\lambda \lambda 3346,3426$, the dashed line is for [Ne IV] $\lambda \lambda 1602$, 4724, while the long-and-shortdashed line is for [Ne III] $\lambda \lambda 3869,3968$.

absorption, corresponding to $E(B-V)=0.15$ mag according to the Munari \& Zwitter (1997) method. Similarly, measuring the EW of the separate components and adding the individual $E(B-V)$, we obtain $E(B-V)=0.16$ mag. However, these $E(B-V)$ may be only lower limits, since the interstellar neutral sodium could have condensed into dust and be depleted in the gas phase. Therefore, for our reddening estimate we also turned to the neutral hydrogen column density. This can be derived by modeling the interstellar Ly $\alpha$ absorption in the day 837 spectrum $^{9}$ by assuming different column densities (Figure 3, top panel). The best Ly $\alpha$ match is obtained for neutral hydrogen column densities of $\sim 7.2 \times 10^{21} \mathrm{~cm}^{-2}$ corresponding to $E(B-V)=0.15 \mathrm{mag}$, in agreement with the NaID estimate. We additionally checked that the derived column density is consistent with that computed by integrating the H I $21 \mathrm{~cm}$ emission in the LAB and GASS maps (Kalberla

\footnotetext{
9 The comparison with previous-epoch spectra guaranteed that the Lyo absorption was purely of interstellar origin.
}

et al. 2005; Kalberla \& Haud 2015) in the velocity range corresponding to the NaID interstellar absorption profile $\mathrm{e}^{10}$ (Figure 3, bottom panel). We adopt $E(B-V)=0.15 \mathrm{mag}$ and the Cardelli et al. (1989) extinction curve when dereddening our data in the following analysis and measurements.

\subsection{Electron Density}

We computed the electron density, $n_{e}$, and derived its variation over time. Despite the optimal wavelength coverage of our spectra, we are often impeded in the use of the classic diagnostics because of the heavy blends or small gaps in spectral coverage. We could not use the $[\mathrm{Ne} \mathrm{V}]$ and [Ne III] because we do not observe the [Ne V] $\lambda 2975$ emission, and for [Ne III] we are unable to deblend the [Ne III] $\lambda 3342$ from the $\lambda 3346$ owing to superposed emission from O III $\lambda 3341$ (all epochs). We could not use the [N II] diagnostic even when the

\footnotetext{
${ }^{10}$ As a consistency check, the UV resonance lines O I $\lambda 1302$, Si II $\lambda \lambda 1260$, 1526, Al II $\lambda 1670$, and C I $\lambda 1561$ were also measured.
} 

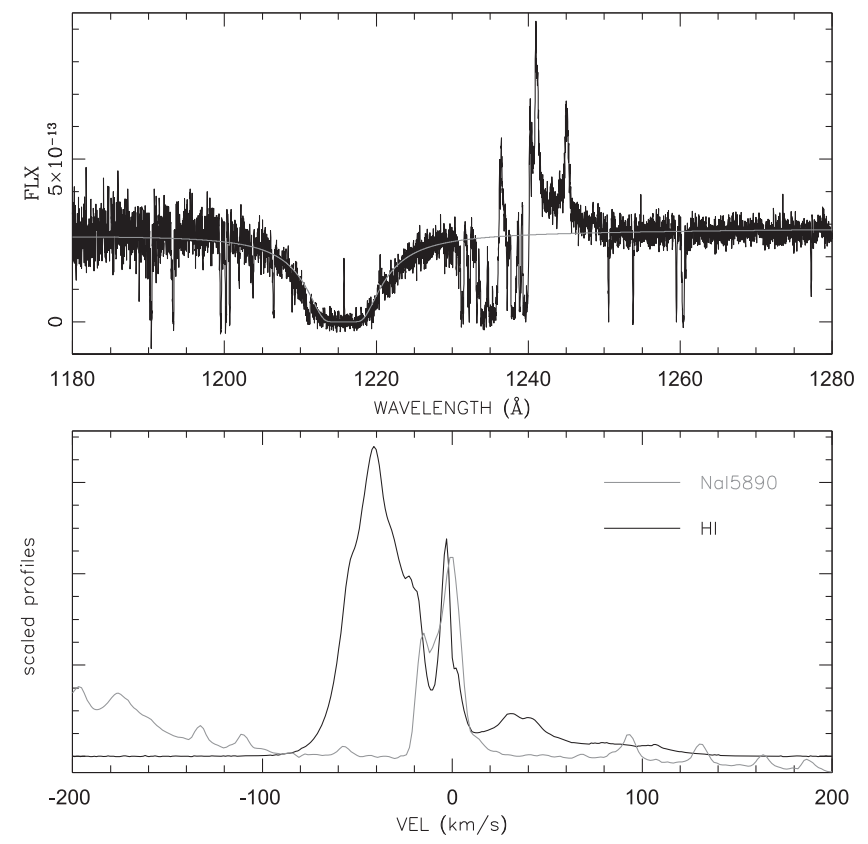

Figure 3. Top panel: Voigt profile fit of the Ly $\alpha$ interstellar absorption in the day 837 V1369 Cen spectrum. Bottom panel: comparison of the $\mathrm{H}$ I line profile from the GASS survey with the $\mathrm{Na}$ I interstellar absorption as seen against V1369 Cen's maximum and early-decline spectra. Note that the $\mathrm{Na}$ I absorption has been inverted for an easier comparison.

$\lambda \lambda 6548,6583$ doublet was separable from $\mathrm{H} \alpha$ because the [N II] $\lambda 5755$ emission line falls in the gap of the UVES red CCD mosaic in the VLT observations. Instead, we used the [O III] diagnostic, deblending the $\lambda 4363$ from the $\mathrm{H} \gamma$ line (by scaling $\mathrm{H} \beta$ to the blue wing of $\mathrm{H} \gamma$ as in our previous studies).

Unlike previous analyses in the literature, our method does not use the integrated line flux. We computed the electron density for each velocity resolution element. The advantage is that of retaining information about the ejecta projected geometry and kinematics and revealing different local conditions. We plot in Figure 4 the electron density in velocity space at the four epochs of Table 1, as derived from the [O III] emission lines. The plot shows how the electron density decreases from $\sim 10^{7} \mathrm{~cm}^{-3}$ to less than $10^{6} \mathrm{~cm}^{-3}$ between days 242 and 835, at least for the low-velocity $\left(\left|v_{\text {rad }}\right| \leqslant 800 \mathrm{~km} \mathrm{~s}^{-1}\right)$ portion of the ejecta. The high-velocity gas (i.e., $\left|v_{\text {rad }}\right| \geqslant 800 \mathrm{~km} \mathrm{~s}^{-1}$ ) in [O III] is too weak to allow density computations. The low-velocity gas, however, is not uniform in density, and the portions between $-700 \mathrm{~km} \mathrm{~s}^{-1} \leqslant v_{\text {rad }} \leqslant$ -500 and $400 \mathrm{~km} \mathrm{~s}^{-1} \leqslant v_{\text {rad }} \leqslant 600 \mathrm{~km} \mathrm{~s}^{-1}$ have somewhat higher density than the line "center" $\left(-500 \mathrm{~km} \mathrm{~s}^{-1}<v_{\text {rad }}<\right.$ $400 \mathrm{~km} \mathrm{~s}^{-1}$ ). The contrast increases from day 242 to day 835 up to a factor of 2 . The innermost portion of the ejecta follows the density decrease expected for ballistic expansion (i.e., $n_{e} \propto t^{-3}$ ), while the intermediate portion of the ejecta follows a less steep decrease in time $\left(n_{e} \propto t^{-2.4}\right.$; see the bottom right panel of Figure 4). This does not imply that the ejecta is following different expansion laws in different portions, but that the intermediate-velocity portion of the ejecta is not yet in the collisionless limit.

In addition, the profile of the UV transitions in the last epoch (day 837) has evolved in such a way that the two doublets C III] $\lambda \lambda 1906,1908$ and N IV] $\lambda \lambda 1483,1486$ are no longer blended and also can be used as density diagnostics. This is a first for novae, although the diagnostic is standard for $\mathrm{H}$ II regions, planetary nebulae, and symbiotic stars. Note that these ratios are independent of reddening. Figure 5 shows (left panels) V1369 Cen C III] and N IV] line profiles and, for comparison (right panels), the same transitions in the last spectrum of V339 Del (day 866). The doublets are blended in V339 Del, even at the last epoch, and the line profile shows an obvious asymmetry of the ejecta. We will return to this in Section 6 when discussing the geometry.

Nussbaumer \& Schild (1981) plot the N IV] line ratio for pure recombination as a function of the electron density for a range of temperatures. We measured the N IV] line ratio for the blue and red peaks separately and ascribe any difference to noise. We obtain an average line ratio of $0.52 \pm 0.06$ so that $n_{e} \simeq 5.2 \times 10^{5} \mathrm{~cm}^{-3}$, compared with the average value of $5.6 \times 10^{5} \mathrm{~cm}^{-3}$ from the [O III] density diagnostic at the blue and red peaks. Note that the [O III] and N IV] peaks yield both comparable densities and density decline rates (as computed for the N IV from the last two epochs). The $\mathrm{C}^{2+}$ recombination coefficient and line ratio as a function of density have been computed by Nussbaumer \& Schild (1979). We measured the average flux ratio $0.30 \pm 0.05$ from the blue and red peaks of the C III] transition in the day 837 spectrum (after a boxcar smoothing of 15 resolution elements). Assuming an electron temperature $T_{e}=10,000 \mathrm{~K}$, we derive from Nussbaumer \& Schild (their Figure 5(a)) $n_{e} \sim 1.4 \times 10^{5} \mathrm{~cm}^{-3}$.

\subsection{Abundance Ratios from Absorption Features}

A striking important result can be obtained from the comparison of the discrete absorption features for $\mathrm{N} \mathrm{V} \lambda \lambda 1238$, 1242 and $\mathrm{C}$ IV $\lambda \lambda 1548,1550$. In V1369 Cen we observe a significant number of coincident (in velocity) $\mathrm{N} \mathrm{V}$ and $\mathrm{C}$ IV absorptions, whose EWs were used to obtain the $\mathrm{N}^{4+} / \mathrm{C}^{3+}$ column density ratio as follows:

$(\mathrm{N} / \mathrm{C})_{v_{\mathrm{rad}}}=\left(\mathrm{EW}_{\mathrm{N}} / \mathrm{EW}_{\mathrm{C}}\right)\left(\lambda_{\mathrm{C}} / \lambda_{\mathrm{N}}\right)^{2}\left(f_{\mathrm{C}} / f_{\mathrm{N}}\right) \sim 1.8\left(\mathrm{EW}_{\mathrm{N}} / \mathrm{EW}_{\mathrm{C}}\right)$,

where $\lambda$ is the rest wavelength and $f$ is the oscillator strength. The common velocity components were at $-1840,-1570$, -1345 , and $-1150 \mathrm{~km} \mathrm{~s}^{-1}$ (see Figure 6), and the derived $\mathrm{N}^{4+} / \mathrm{C}^{3+}$ column density ratio was $270,200,233$, and $>120$, respectively, with an uncertainty of about $10 \%$. Thus, excluding the lower limit and assuming that the measured resonance lines represent the dominant ion state, the ratio of nitrogen to carbon was $\geqslant 200$. The solar ratio is 0.31 (Asplund et al. 2009), implying that V1369 Cen was enormously enhanced in nitrogen relative to carbon. The ratio is more extreme than published nucleosynthetic yields of the TNR of massive white dwarfs (WDs; e.g., Downen et al. 2013; Casanova et al. 2016; Starrfield et al. 2016).

A similar analysis applied to T Pyx and V339 Del produced different values. In particular, we found an $\mathrm{N} / \mathrm{C}$ ratio in the range of $[1,6]$ for the case of V339 $\operatorname{Del}^{11}$ and $\mathrm{N} / \mathrm{C} \simeq 2.3 \pm 0.2$ for $\mathrm{T}$ Pyx. In both cases, the values are not as different relative to the solar value as for V1369 Cen, and they are compatible with theoretical TNR predictions.

Note that in all cases no information is available for the isotopic composition of the clumps. Although consistent with the general expectation for the explosive yields, these

\footnotetext{
11 Note that the value is quite uncertain, since V339 Del absorption features remained less distinct even in the last epoch.
} 

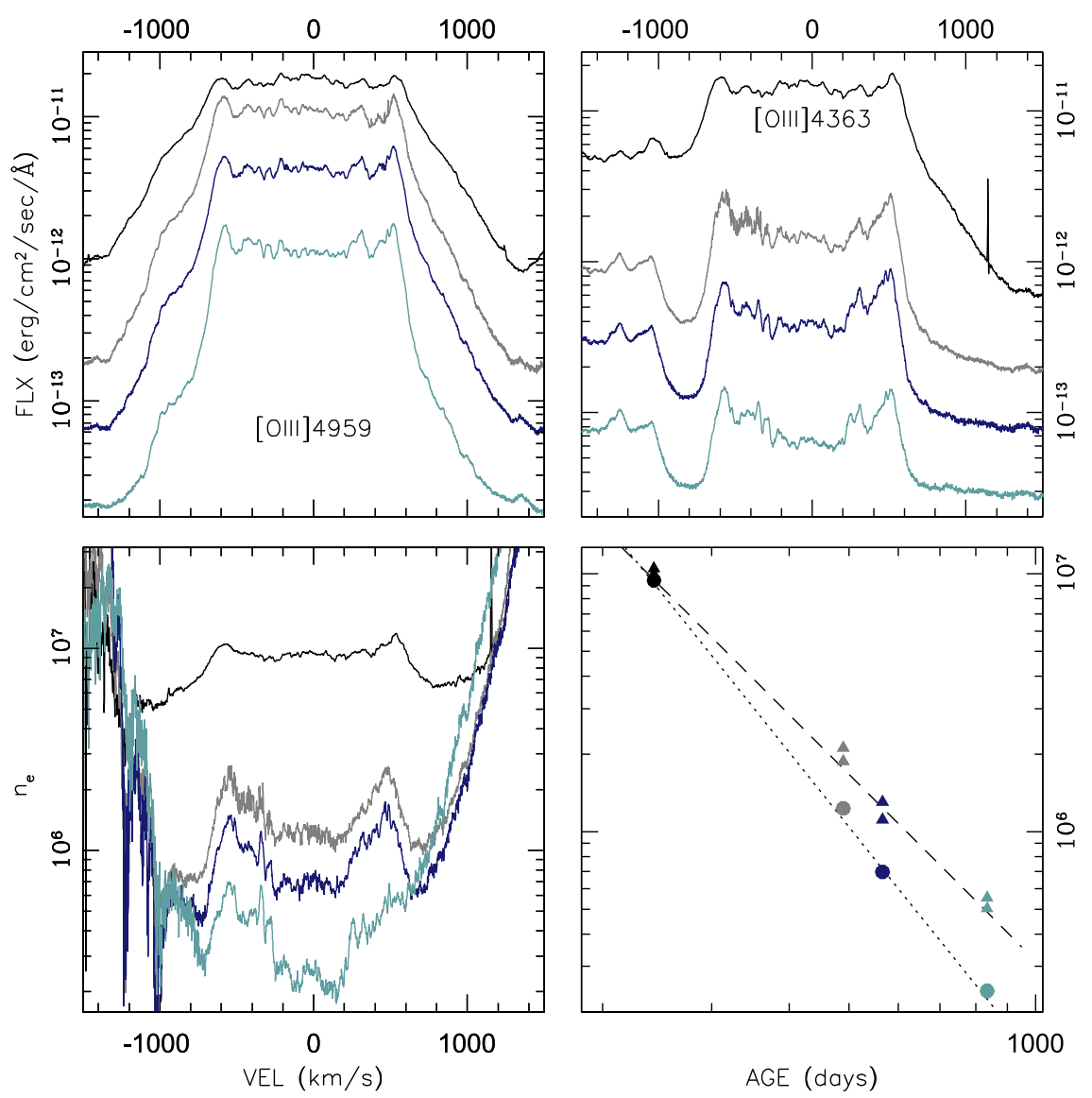

Figure 4. V1369 Cen [O III] line profiles (top panels) and electron density as derived from the [O III] emission diagnostic: in velocity space at various epochs (bottom left panel) and as a function of time (bottom right panel). Note that both fluxes (top two panels) and densities (bottom two panels) are in log scale to enhance the contrasts. Different color indicates different epoch, as can be evinced from the bottom right panel. In the bottom right panel filled circles indicate the average density as measured in the velocity interval -100 to $100 \mathrm{~km} \mathrm{~s}^{-1}$, while the triangles indicate the average density at the two peaks centered at -600 and $500 \mathrm{~km} \mathrm{~s}^{-1}$.

abundance ratios cannot place precise constraints on the thermodynamic condition at the peak of the TNR.

\subsection{Filling Factor and Distance}

The first STIS spectra were taken at the highest echelle resolution when the nova was especially bright. This affords the best interstellar absorption line profiles, and we have used the O I $\lambda 1302$, Si II $\lambda 1260, \mathrm{C}$ I $\lambda 1561$, and $\mathrm{Al}$ II $\lambda 1670$ lines to provide a range of ionizations and line strengths. Except for $\mathrm{O}$ I, all are strong but unsaturated. The interval covered by the profiles was $-11 \mathrm{~km} \mathrm{~s}^{-1} \leqslant v_{\text {rad,helio }} \leqslant 14 \mathrm{~km} \mathrm{~s}^{-1}$ with a mean velocity of $1.4 \mathrm{~km} \mathrm{~s}^{-1}$ (weighted flux distribution), corresponding to $v_{\text {rad,LSR }}=-2.4 \mathrm{~km} \mathrm{~s}^{-1}$ in the local standard of rest (LSR). Using the IAU standard Oort constants and distance to the Galactic center, this velocity implies a distance $1.8 \leqslant D$ $(\mathrm{kpc}) \leqslant 2.4$. We adopt $2 \mathrm{kpc}$ in what follows. This is also consistent with the extinction. The distance is independent of any maximum magnitude versus rate of decline (MMRD) assumptions. As we discussed, V1369 Cen did not resemble either V959 Mon (or other ONe classical novae) or V339 Del, so we cannot scale the spectra relative to previously observed exemplars.

For mass determination, we take advantage of the multiple determinations of the $n_{e}$ from the last-epoch optical and UV spectrum (day 835/837). The measured $\mathrm{H} \beta$ flux was $F(\mathrm{H} \beta)=1.47 \times 10^{-12} \mathrm{erg} \mathrm{s}^{-1} \mathrm{~cm}^{-2}$. With a reddening $E(B-V)=0.15$ applied and for a distance of $2 \mathrm{kpc}$, the line luminosity was $L(\mathrm{H} \beta)=1.2 \times 10^{32}(D / 2 \mathrm{kpc})^{2} \mathrm{erg} \mathrm{s}^{-1}$. The range of $n_{e}$ obtained from the $\mathrm{C}$ III] $\lambda 1908$, N IV] $\lambda 1486$, and [O III] nebular lines is $1<\left(n_{e} / 10^{5}\right) \mathrm{cm}^{-3}<6$, giving a predicted range $0.5<L(\mathrm{H} \beta) /\left(10^{35} \mathrm{erg} \mathrm{s}^{-1}\right)<12$ with a choice of $v_{\max }=2500 \mathrm{~km} \mathrm{~s}^{-1}$. Although this is a broad range, other quantities are fairly well constrained: the solid angle of the aspherical ejecta as provided by the bipolar models (see Section 6.2) is $(\Delta \Omega / 4 \pi) \approx 1 / 3$, and the filling factor is in the range of $0.1<f<0.2$. Combined with the electron density, the ejecta mass resulted in approximately $(1.1 \pm 0.4) \times 10^{-4} M_{\odot}$ adopting a solar $\mathrm{He} / \mathrm{H}$ ratio. Although some novae appear to have either hydrogen deficiencies or enhanced helium abundances (e.g., José \& Shore 2008; Schwarz 2014), the electron density is obtained directly from the plasma diagnostics and not from the $\mathrm{H} \beta$ flux, which was used only to derive the filling factor. The filling factor scales as $f \sim[1+n(\mathrm{He}) / n(\mathrm{H})]^{-1}$, and the mass scales as $(\mu / 1.6) f$. The independent determination of the helium abundance is only possible with detailed modeling of the recombination and ionization structure of the ejecta and applies only during the photoionization-dominated stage before SSS turnoff. We note, alternatively, that the mass scales as $M_{e j} \sim v_{\max }^{-3 / 2} D n_{e}[E(B-V)]^{1 / 2}$.

This mass is higher than we found for V339 Del $\left((2-3) \times 10^{-5} M_{\odot}\right.$; Shore et al. 2016) and V959 Mon ( $\lesssim 6 \times 10^{-5} M_{\odot}$; Shore et al. $2013 \mathrm{a}$ ), but not excessively so, and is around the values expected from the models of the TNR (Downen et al. 2013). 

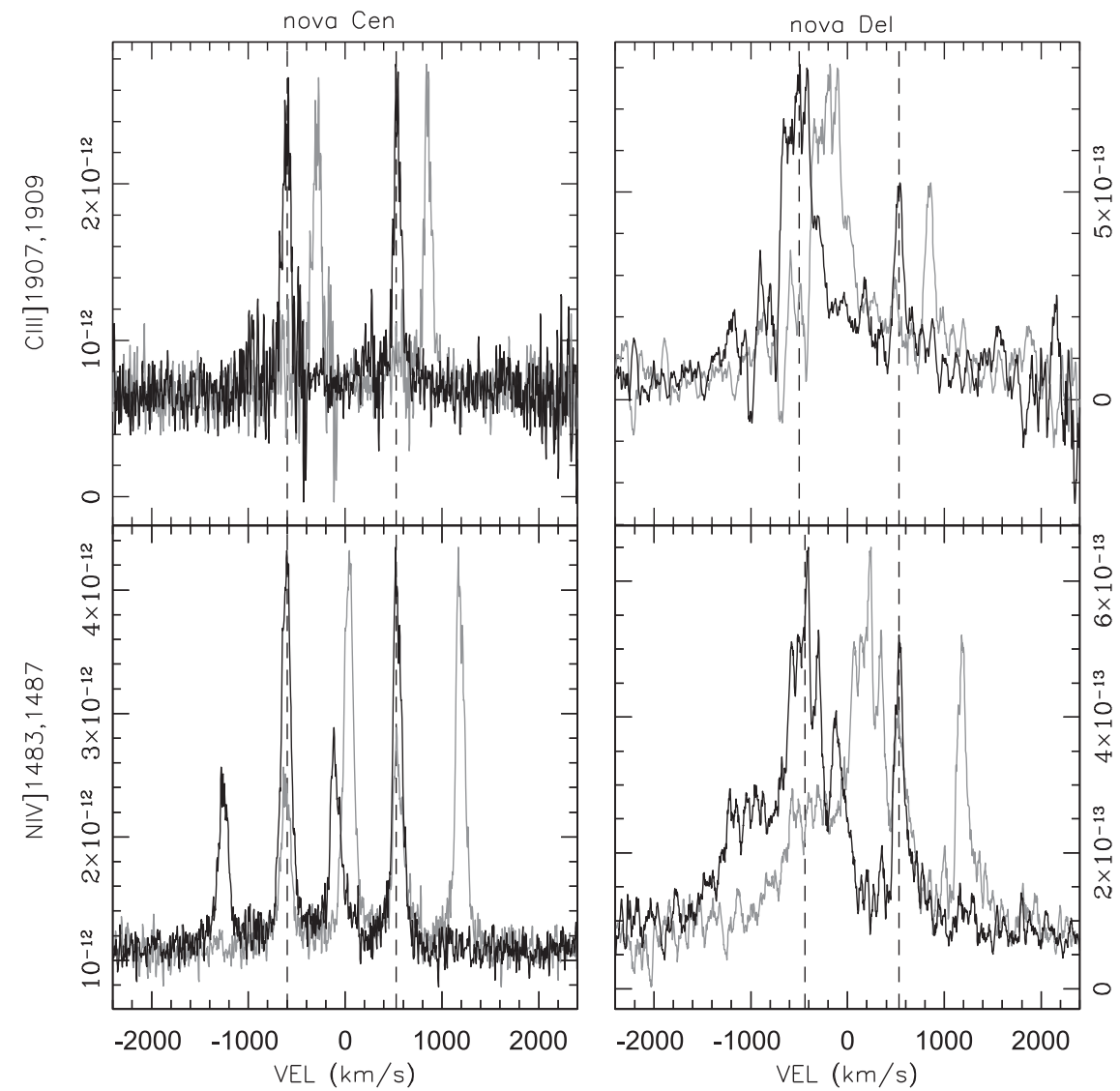

Figure 5. V1369 Cen (left) and V339 Del (right) C III (top) and N IV (bottom) line profiles at their last epoch. The black color is for C III] $\lambda 1908$ (N IV] $\lambda 1486$ ), while the gray is for C III] $\lambda 1906$ (N IV] $\lambda 1483$ ). Vertical dashed lines mark the blue and red peaks of the emission line. V339 Del asymmetric profiles indicate geometrical (at the substructure level) asymmetry. Density inhomogeneity is also possible but cannot be verified from the lines themselves because the doublets are blending.

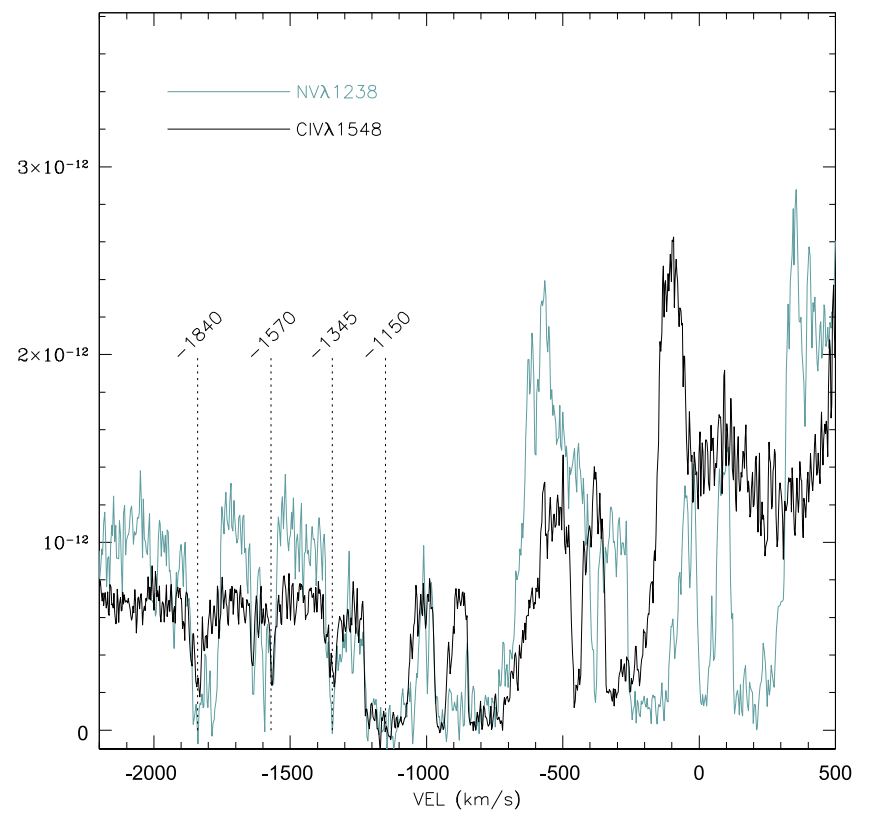

Figure 6. Absorption structures observed in the day 837 spectrum for the transitions $\mathrm{N}$ V $\lambda \lambda 1238,1242$ and $\mathrm{C}$ IV $\lambda \lambda 1548,1550$. Vertical dotted lines mark the approximate wavelength of the absorption components used for the $\mathrm{N} / \mathrm{C}$ ratio computation. The $y$-axis is flux in erg cm $\mathrm{cm}^{-2} \mathrm{~s}^{-1} \AA^{-1}$.

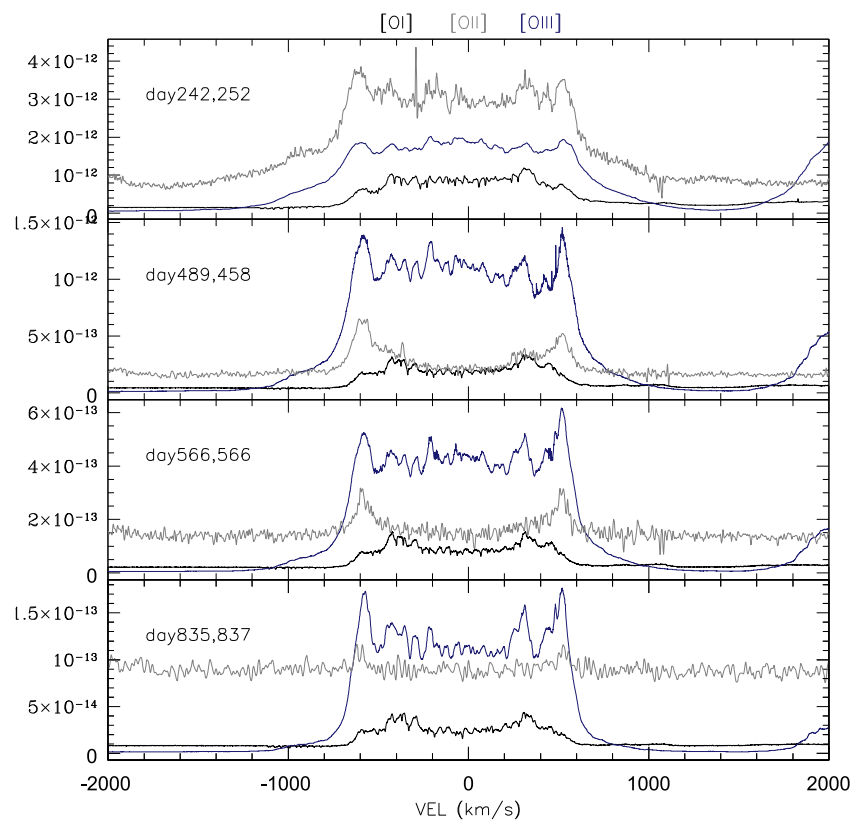

Figure 7. V1369 Cen ionization structure as evinced from the nebular lines [O III] $\lambda 4959$, [O II] $\lambda 2470$, and [O I] $\lambda 6300$ at the four epochs. Note that [O III] $\lambda 4959$ has been scaled by a factor of 0.1 , while [O II] $\lambda 2470$ has been scaled by a factor of 0.4 . 

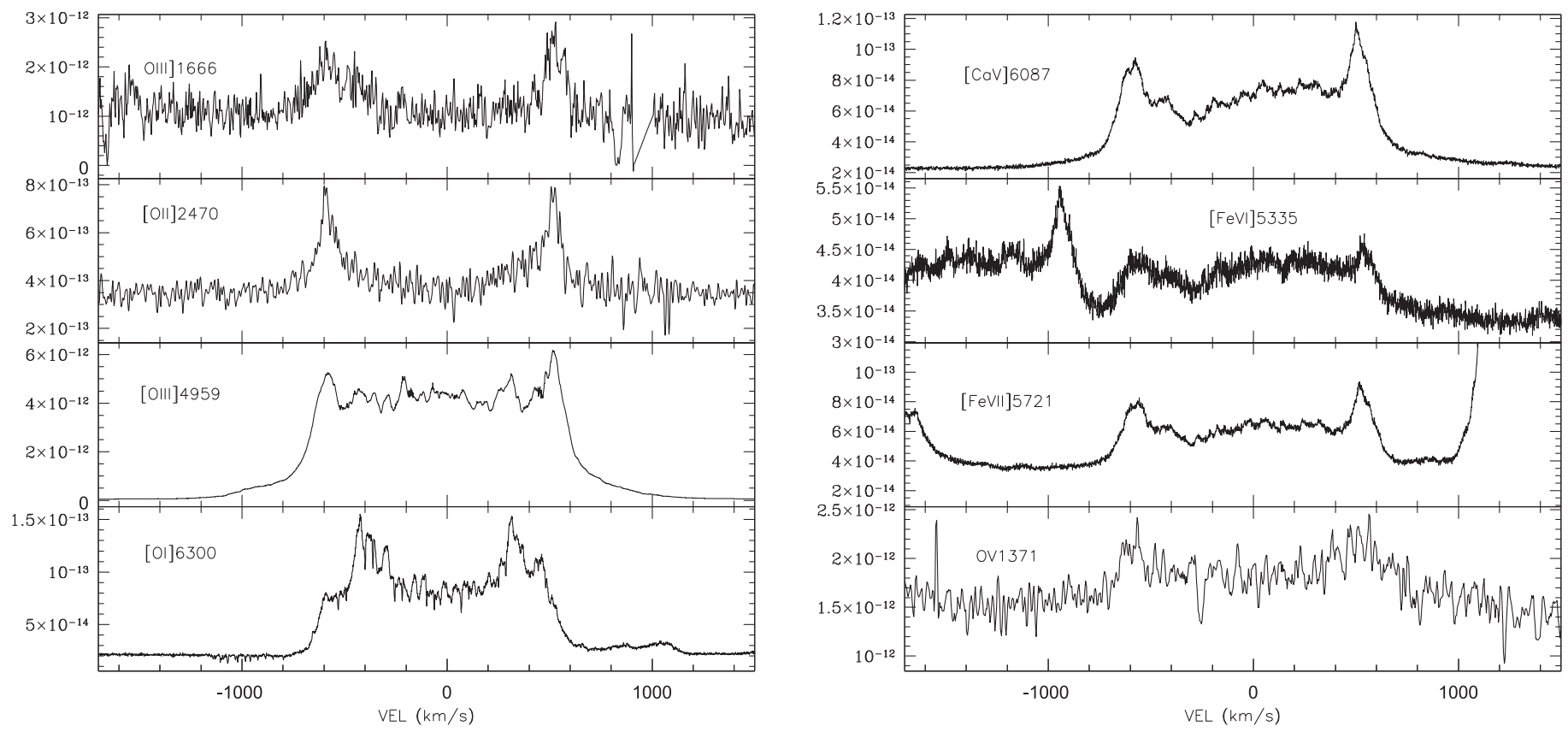

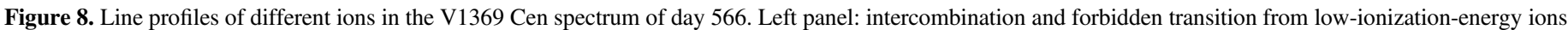

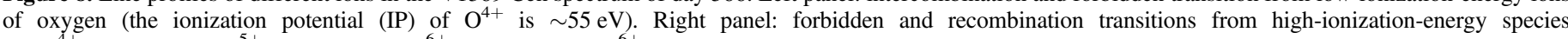
$\operatorname{IP}\left(\mathrm{Ca}^{4+}\right) \simeq 67 \mathrm{ev}, \operatorname{IP}\left(\mathrm{Fe}^{5+}\right) \simeq 76 \mathrm{eV}, \operatorname{IP}\left(\mathrm{Fe}^{6+}\right) \simeq 100 \mathrm{eV}, \operatorname{IP}\left(\mathrm{O}^{6+}\right) \sim 113 \mathrm{eV}$

\section{Ejecta Geometry and Ionization Structure}

In this section we analyze the ejecta ionization structure and infer the ejecta geometry. Note that the ionization structure is a key component in our work since it serves to constrain a selfconsistent scenario.

There are two ways of looking at the ejecta structure in general and their ionization structure in particular: (1) the emission lines and (2) the absorption components. We explain these in turn.

\subsection{Analysis of the Emission Lines}

One must never forget that the emission-line profile is the result of a line-of-sight velocity projection effect. For optically thin gas, we see everything that has a sufficient emission measure in a given radial velocity bin, so we measure the sum of the contributions from individual clumps, each of which may have different density conditions. Hence, even the density in velocity space, as in Figure 4, is an average per velocity bin. The diverse local conditions of the clumps imply that different parts of the ejecta might develop differently depending on their different outward velocity relative to the WD. However, once each clump is in pure recombination for a given ion, the line profile is "frozen" and the line flux simply decreases with time with no change in $v_{\text {rad }}$. With this in mind, we can compare lines produced by different mechanisms (e.g., recombination, collisions exciting a metastable level, or intercombination transitions) and by different ions of the same element. These comparisons yield the boundaries of the distribution of various ions, i.e., the ionization structure, and help disentangle abundance from ionization effects in the observed line profiles.

To illustrate this for the V1369 Cen ejecta, we compare (1) the temporal evolution of three forbidden transitions of oxygen from different ionization stages $\left(\mathrm{O}^{0}\right.$ to $\left.\mathrm{O}^{2+}\right)$; (2) the different profiles of recombination, intercombination, and forbidden transitions from low- and high-ionization energy ions at epoch $\sim 566$ days; and (3) the profiles of recombination lines from different ions at day 835 .

The evolution of the oxygen ions is shown in Figure 7. Since this treats a single element in various ionization stages, it shows the development of the ionization structure unbiased by abundance. It is interesting to note here that in the first epoch (day 242/252), [O II] and [O III] both have very pronounced wings $\left(800 \mathrm{~km} \mathrm{~s}^{-1}<\left|v_{\text {rad }}\right|<1600 \mathrm{~km} \mathrm{~s}^{-1}\right)$, which quickly disappear for the [O II] transitions but persist (although weakened) in [O III]. Hence, in that velocity range the densities are low enough that $\mathrm{O}^{2+}$ no longer recombines to $\mathrm{O}^{+}$as easily. Oxygen exists mostly in the double ionized state, which is excited from the ground level by collisions (and the collisional excitation cross section is larger than the collisional recombination cross section). Similarly, the neutral O is confined to higherdensity regions that correspond to the lower-velocity bins. By projection, the peak intensity of the [O I] transition occurs at $-400 \mathrm{~km} \mathrm{~s}^{-1}$ and $+300 \mathrm{~km} \mathrm{~s}^{-1}$, while the [O III] emissions have their peak intensity at $-600 \mathrm{~km} \mathrm{~s}^{-1}$ and $+550 \mathrm{~km} \mathrm{~s}^{-1} \cdot{ }^{12}$ At those velocities the [O I] shows only a weak shoulder. This implies that the $\mathrm{O}^{2+}$ concentrates, on average, at larger velocities with respect to $\mathrm{O}^{0}$, i.e., the $\mathrm{O}^{2+} / \mathrm{O}^{0}$ ratio increases at higher velocity. The lowest $v_{\text {rad }}$ portion of the emission lines (velocity range $-300 \mathrm{~km} \mathrm{~s}^{-1}<v_{\text {rad }}<200 \mathrm{~km} \mathrm{~s}^{-1}$ ) shows relatively higher flux in the [O III] transition than in the [O II] and [O I], especially at epochs 458 and 566, indicating that there are projected low-velocity regions where $\mathrm{O}^{2+}$ does not recombine. In other words, the oxygen is mostly in the $\mathrm{O}^{2+}$ state everywhere across the ejecta but is relatively more abundant-compared to $\mathrm{O}^{+}$and $\mathrm{O}^{0}$-in the outer portion of the ejecta (those with the higher velocity), where the density must be lower for the recombination rate to be consequently lower. The $\mathrm{O}^{+}$tracer, [O II] 22470 , disappears earlier (at day 837 it is almost completely gone) because of the higher energy of its upper level.

\footnotetext{
${ }^{12}$ Note also that recombination lines display peaks at those same velocities.
} 


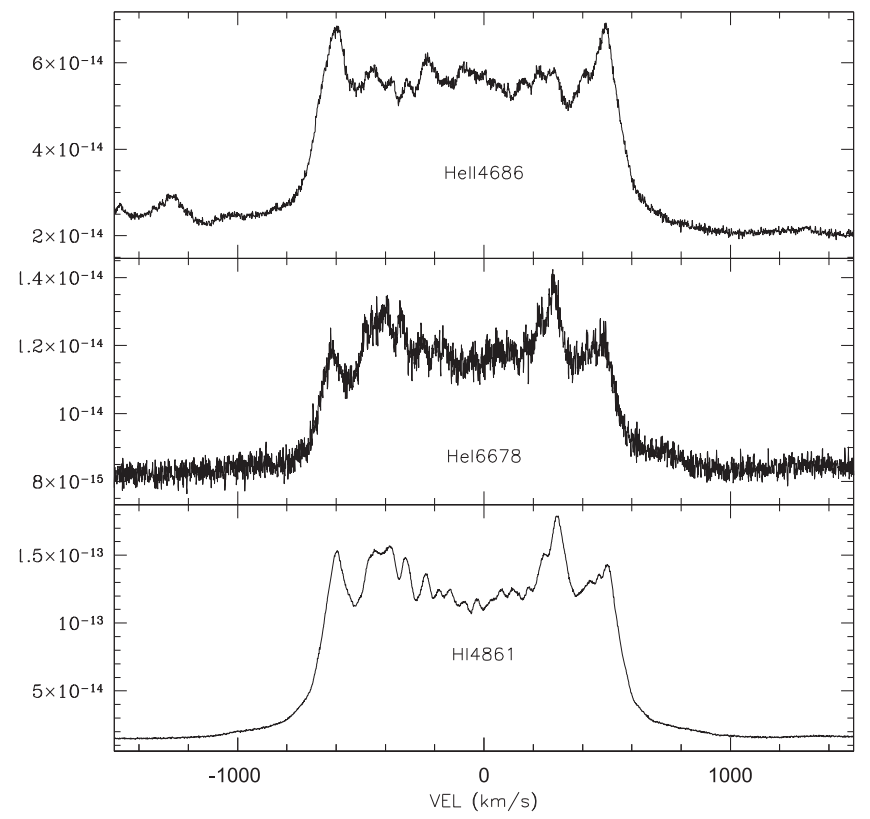

Figure 9. V1369 Cen $\mathrm{H}$ and He recombination line on day 835. On the $y$-axis of the plot, fluxes are in erg $\mathrm{cm}^{-2} \mathrm{~s}^{-1} \AA^{-1}$.

Figure 8 shows forbidden, intercombination, and recombination transitions from different ions observed on day 566. In particular, the left panel compares line profiles from lowenergy oxygen ions, including the $\mathrm{O}$ III] $\lambda 1666$ line, which is an intercombination transition and shows where $\mathrm{O}^{3+}$ recombines to $\mathrm{O}^{2+}$. The transition shows emission at the -600 and $+500 \mathrm{~km} \mathrm{~s}^{-1}$ peaks and is undetectable everywhere else, i.e., the emission measure is larger where the density is not too low to prevent the $\mathrm{O}^{3+}$ recombination and not too high to favor the recombination of lower ions. The right panel compares forbidden and recombination transitions from high-energy ions $(67 \mathrm{eV} \leqslant \mathrm{IP} \leqslant 113 \mathrm{eV}$, where IP is the ionization potential), showing that their profiles are similar, i.e., the ions are cospatial. We note that the $\mathrm{OV} \lambda 1371$ line and similar recombination transitions (e.g., N IV $\lambda 1718$ ) are frozen since high-energy ionizing photons are no longer dominant. The highest-energy ions provide information about the spectral energy distribution of the underlying photoionizing source: the detection of $\mathrm{OV}$ and [Fe VII] lines and the lack of [Fe X] constrain the SSS (when on) to temperatures $>100-130 \mathrm{eV}$ and $<200 \mathrm{eV}$.

Finally, we show in Figure 9 the profiles of the recombination transitions He II $\lambda 4686$ and the singlet He I $\lambda 6678$, together with $\mathrm{H} \beta \lambda 4861$ at day 835. The two He lines show the distribution of singly and doubly ionized $\mathrm{He}$; since $\mathrm{H}$ is the most abundant element, $\mathrm{H} \beta$ shows the projected geometry of the ejecta. The He I profile resembles the [O I] line, but with more pronounced peaks at -400 and $+300 \mathrm{~km} \mathrm{~s}^{-1}$. In contrast, He II has peaks at -600 and $+500 \mathrm{~km} \mathrm{~s}^{-1}$ that are very similar to [O III], although the two He II peaks have greater contrast relative to the rest of the line than those in the [O III] line profile. The similarity is not surprising, since $\mathrm{He}$ II and [O III] trace the distribution of the highly ionized gas, while He I and [O I] trace the neutral gas. The individual different contrasts in each line result from the fact that recombination lines have much higher transition probability than forbidden lines, and these also depend on their collisional cross section and the electron energy distribution for the population of their upper level.

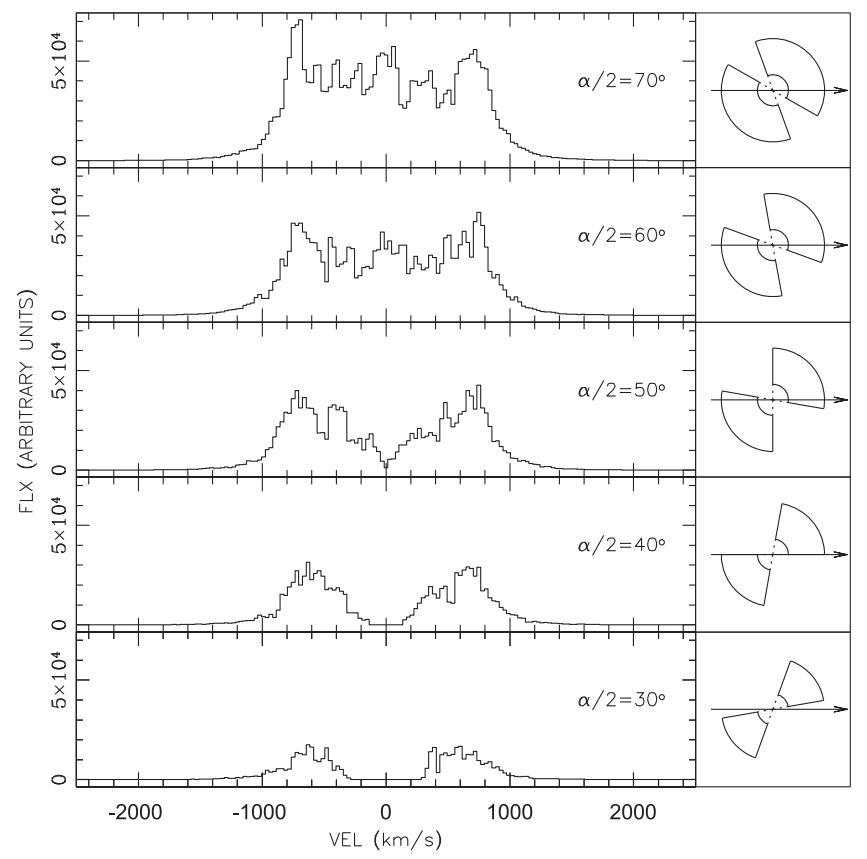

Figure 10. Line profiles produced by a biconical ejecta as simulated by Shore et al. (2013b). In all cases we assume (1) an inclination of $40^{\circ}$ of the observer with respect to the bi-cone axis, and (2) an ejecta thickness of $0.7 \times R$, where $R$ is the maximum ejecta radius, and that the truncated cone is filled in. The simulation differs in the opening angle $\alpha$ of the cone, indicated in the figure itself. A $2 \mathrm{D}$ sketch of the corresponding ejecta geometry is indicated on the right of each profile. The arrow points to the observer.

\subsection{Monte Carlo Simulation of the Emission Lines}

In this section we apply the Monte Carlo simulation already used for T Pyx, V959 Mon, and V339 Del, to reproduce the observed line profiles in the V1369 Cen spectra. The code uses random particles confined in a biconical geometry to reproduce the gross characteristics of the line profiles. The random particles have a linear velocity law within a range matching the observations, constant mass, and emissivity corresponding to the evolutionary stage of the nova (recombination). Given the frozen state of the ejecta, it would be physically inconsistent to adopt any photoionization code to reproduce the observed line profile. Photoionization codes assume equilibrium (which is not physically appropriate) and do not account for expansion. The simulation reproduces the global line profile but not specific substructures: these result from the simulation noise but correspond to individual clumps overlapping in the projected velocity space, in real ejecta. Modeling of individual substructures would require adopting specific particle distributions, an assumption that is not relevant to the purpose of our work.

A variety of profiles can result from our code, including the most common, so-called "saddle"- and "castellated"-shaped profiles. They are produced by a combination of opening angles, cone thickness, and inclination with respect to the line of sight. Figure 10 shows the effect of the cone opening angle in shaping the line profile for a fixed inclination. Less collimated ejecta produce more "rectangular" (or "flat-top") profiles, while a "saddle"- or V-shaped profile is obtained with more collimated ejecta. In the case of V1369 Cen we could reproduce its "horned" line profiles of width $\sim 1200-1500 \mathrm{~km} \mathrm{~s}^{-1}$ adopting a biconical geometry with opening angle up to $140^{\circ}$, thickness $0.75 R$ (where $R$ is the maximum radius of the ejecta), and maximum expansion velocity $\mathrm{v}=2500 \mathrm{~km} \mathrm{~s}^{-1}$ viewed at an 


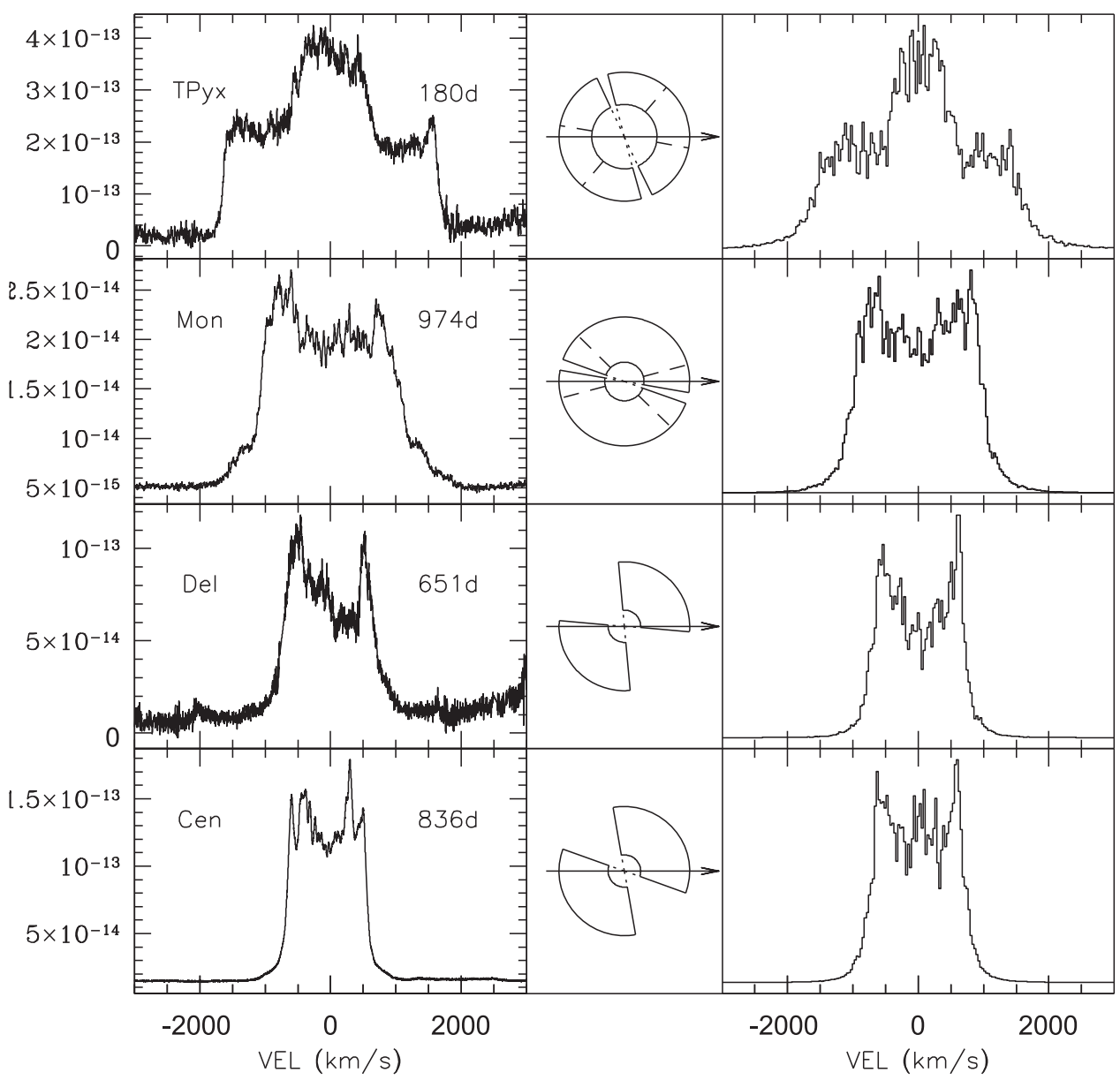

Figure 11. Left column: $\mathrm{H} \beta$ emission line profiles at the last epoch of observation for each of the "panchromatic novae" discussed in this series of publications. The ordinate is flux in units of erg cm $\mathrm{cm}^{-2} \mathrm{~s}^{-1}$. The identification of each nova is on the plot itself, together with the age of the nova (i.e., the epoch of observation in days). T Pyx and V959 Mon spectra have been smoothed by a running boxcar of five points. Middle and right columns: biconical geometry derived for each nova and the corresponding predicted line profile. Dashed lines in the model geometry from T Pyx and V959 Mon indicate a hollow cone (see Shore et al. 2013a, 2013b, for more details).

inclination of $\sim 40^{\circ}$. Figure 11 displays the $\mathrm{H} \beta$ profile for $\mathrm{T}$ Pyx, V959 Mon, V339 Del, and V1369 Cen, together with the biconical geometry that best mimics their profile and the corresponding Monte Carlo simulation predicted profile. The figure shows the gross similarities and the specific differences characteristic of each ejecta. The emission lines of a given nova can be broader or narrow and more or less rectangular in shape. They can also display extended wings as in the case of T Pyx. All trace back to the specific characteristic of the biconical ballistic ejecta.

Note that the simulations are not fitting each profile but providing a tool for interpreting the data. In particular, (1) for each nova, the opening angle varies with the transition as different ions have different structures (e.g., [O III] typically suggests a slightly wider opening angle than [O I] given that the most external regions are less dense and, therefore, more highly ionized; see Section 6.1); (2) there might be degeneracies in the simulation (see Shore et al. 2013b); (3) the line widths and the emission measure decreases with time; and (4) the simulations produce globally symmetric profiles. Asymmetries such as those observed in V339 Del at each epoch and in all transitions (see Shore et al. 2016) likely reflect intrinsic characteristics of the ejecta whose origins remain to be investigated. Hence, the takeaway result from the simulation is that all of the gross features of the observed profiles for each nova can be reproduced by a biconical geometry with relatively wide opening angles and relatively large thickness. Features resembling rings, equatorial belts, and polar caps also result from projection effects of the biconical geometry onto the sky plane (see, e.g., Figure 10 in Shore et al. 2013b; Figures 16 and 20 in Shore et al. 2013a). Thus, bipolar ejection seems to be common to all novae without requiring additional specially tailored structures such as equatorial rings or jets.

\subsection{Analysis of the Absorption Features}

For the absorption structures, we see only what is along the line of sight, i.e., any structure that is lying between us and the hot receding surface of the WD. During maximum and early decline when we see the P Cyg profiles, the absorbing gas and the pseudo-photosphere illuminating it are contiguous since the latter is part of the ejecta themselves (or their bottom layer) and subtends a large solid angle that intercepts numerous structures along the line of sight. As the pseudo-photosphere recedes, the number of intercepted structures (i.e., clumps) decreases. Our ability to detect them depends on the inclination and the ejecta characteristics (given sufficient $\mathrm{S} / \mathrm{N}$ and spectral resolution of the data). Ejecta viewed along the symmetry axis will show a larger number of absorption features. The larger the filling factor, the more absorbing structures are intercepted along the line of sight. The same holds for geometrically thicker ejecta. 


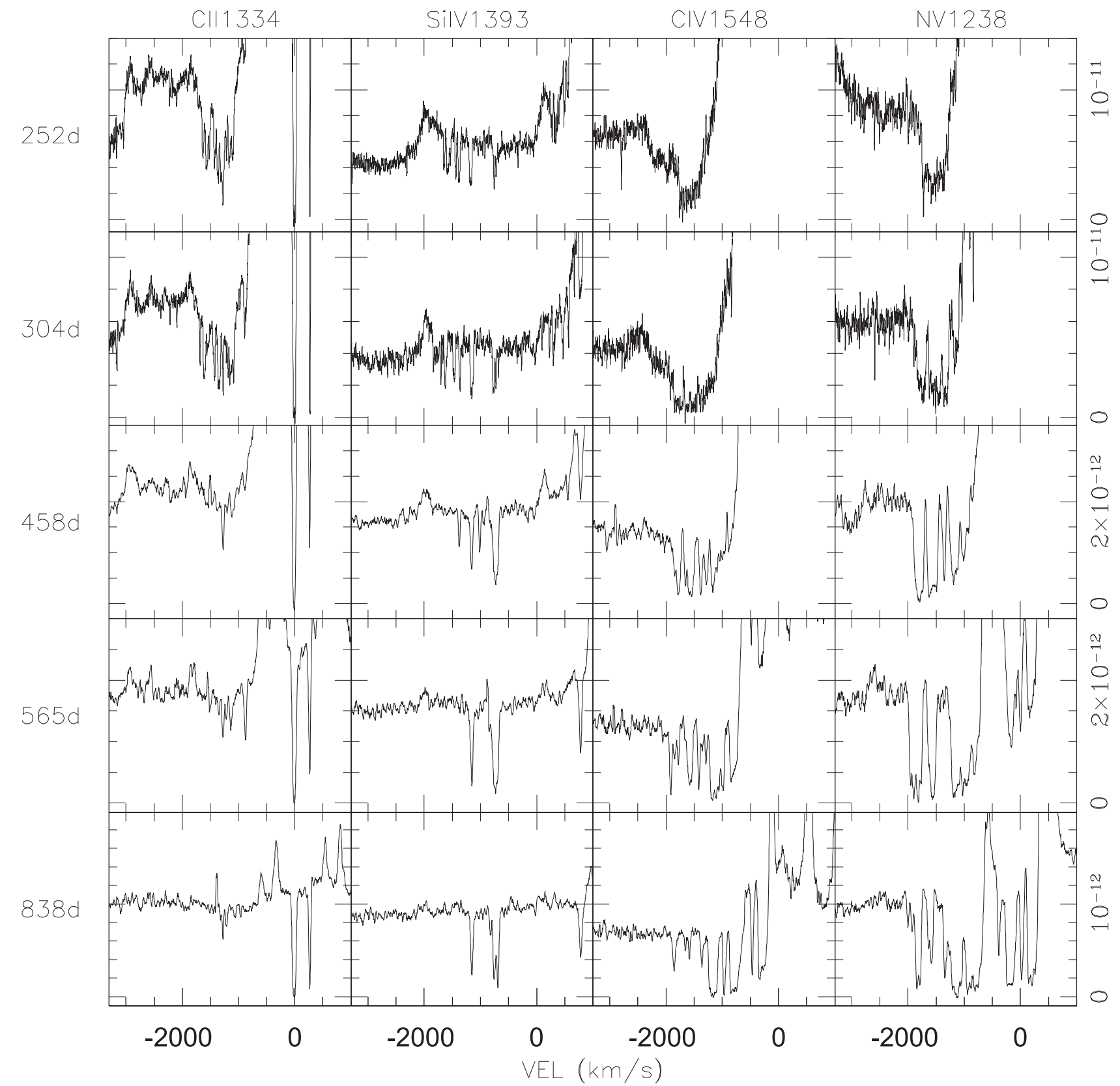

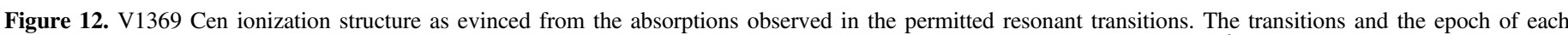
observation are labeled on the figure side (top and left, respectively). On the right axis of the plot, fluxes are in erg cm ${ }^{-2} \mathrm{~s}^{-1} \AA^{-1}$.

V1369 Cen, which is the nova with the best $\mathrm{S} / \mathrm{N}$ and sampling, shows complex absorptions for the four UV resonance transitions C II $\lambda 1334$, Si IV $\lambda 1393$, C IV $\lambda 1548$, and $\mathrm{NV} \lambda 1238$, whose ions have different ionization potential energy: $\sim 11 \mathrm{eV}$ for $\mathrm{C}^{+}, 33 \mathrm{eV}$ for $\mathrm{Si}^{3+}, 47 \mathrm{eV}$ for $\mathrm{C}^{3+}$, and $77 \mathrm{eV}$ for $\mathrm{N}^{4+}$. Figure 12 shows the different absorption patterns produced by each resonant transition at each epoch. Each ion recombines and absorbs where conditions are suitable for it to do so. At early epochs (day 252 in the figure) the densities are sufficiently high that even low ions such as $\mathrm{C}^{2+}$ can recombine to $\mathrm{C}^{+}$(and therefore absorb from the ground states) up to relatively high velocities, i.e., up to the outskirts of the ejecta. Si IV, whose absorptions are superposed on a blending emission in the figure, are distributed up to $-1750 \mathrm{~km} \mathrm{~s}^{-1}$ like C II. In contrast, N V and C IV at that same epoch show absorption troughs (very much like $\mathrm{H}^{0}$ or $\mathrm{Fe}^{+}$ during the early-decline/iron curtain phase): $\mathrm{C}$ and $\mathrm{N}$ are four and five times ionized everywhere, but only in the innermost portion of the ejecta is the density high enough for them to recombine to $\mathrm{C}^{3+}$ and $\mathrm{N}^{4+}$. The number of clumps where this can occur is numerous and blending, and only later on (i.e., from day 458 on) do they appear separately. The range of velocity they cover seems to increase, stretching to both lower and higher velocity limits. The appearance of the low-velocity structures at later epochs is because they are seen against the stellar continuum, while earlier they lie on the emission line and conflate with the emission structures. The structures at the higher velocities (i.e., $\sim-2000 \mathrm{~km} \mathrm{~s}^{-1}$ ) appear as soon as the $\mathrm{C}^{4+}$ and $\mathrm{N}^{5+}$ have recombined to $\mathrm{C}^{3+}$ and $\mathrm{N}^{4+}$, respectively (the recombination time for $\mathrm{N}^{5+} \rightarrow \mathrm{N}^{4+}$ is slow), 
and on day 252 the nitrogen is still 5 times ionized at $v_{\text {rad }}<-1700 \mathrm{~km} \mathrm{~s}^{-1}$. The same holds for $\mathrm{C}$ and its recombination $\mathrm{C}^{4+} \rightarrow \mathrm{C}^{3+}$; see also the end of Section 6.5.

This is consistent with the lack of $\mathrm{C}^{+}$absorptions in the lastepoch spectrum: the majority of $\mathrm{C}$ is trapped in high-ionization states, and the fraction of $\mathrm{C}$ in low-ionization states is not enough to produce a sufficient contrast against the continuum for detection. The particular absorption pattern produced by a given ion also depends on the clumps' alignment with respect to the changing (decreasing) solid angle subtended by the WD photosphere. Note that most of the structures we see are, in fact, blends even at day 837 . The velocity width of individual structures might be as low as $10 \mathrm{~km} \mathrm{~s}^{-1}$, corresponding to radial thickness of only a few percent or less of $R$ (the cone/ ejecta maximum radius).

\subsection{Monte Carlo Simulation of the Absorption Features}

As a novel feature of our approach, we can also check whether the same approximation used to interpret the emissionline profile reproduces the absorption features observed in these late stages. Hence, we used the same Monte Carlo code, with the same set of parameters and geometry as for the emission profiles (see Figure 10 and Section 6.2), to simulate the absorption features. In this case, each density point along a selected line of sight is used to compute the opacity. Screening effects between clumps have been ignored, although superposition is included. This approximation is valid for the late nebular phase spectra, since the velocity separation between clumps along the line of sight is larger than the velocity width of the individual clumps. The result is shown in Figure 13. The figure shows that decreasing the covering factor reduces the number of absorption features, as discussed above.

\subsection{Absorption Features in Other Novae}

The UV resonance line absorptions were first observed in T Pyx (day 834 spectrum; Shore et al. 2013b) in the very late observations of the recurrent nova and only in $\mathrm{N} \mathrm{V} \lambda 1238$ and C IV $\lambda 1548$ absorptions but with only two structures per transitions. This is consistent with a lower mass for the T Pyx ejecta and with a low filling factor. Unfortunately, we do not have late UV spectra of V959 Mon, so we cannot say anything about its absorption structures in the UV resonance transition. The V339 Del absorptions are shown in Figure 14. The overall evolution of the absorptions in the four resonance transitions is very similar to V1369 Cen, but with a few notable differences: (1) The C II $\lambda 1334$ absorptions persist to the end, unlike V1369 Cen, indicating that at similar epochs V1369 Cen reaches lower densities than V339 Del, too low for $\mathrm{C}^{2+}$ to recombine to $\mathrm{C}^{+}$ (the recombination cross section of $\mathrm{C}^{2+}$ is smaller than that of $\mathrm{C}^{3+}$ and hence is favored in higher-density environments). (2) The C IV and NV absorptions in V339 Del are never as fragmented as in V1369 Cen, indicating a different distribution of the clumps along the line of sight (the cumulated EWs are comparable in the two novae).

Global commonalities that we should note are as follows: (i) the velocity ranges spanned by the absorptions are comparable among novae, and (ii) a number of absorption structures persist at the same velocity through all epochs. To better illustrate this, we plot in Figure 15 the absorption structures observed in the low ionization potential metals at early time and in the highionization-energy species at late epochs for all the novae

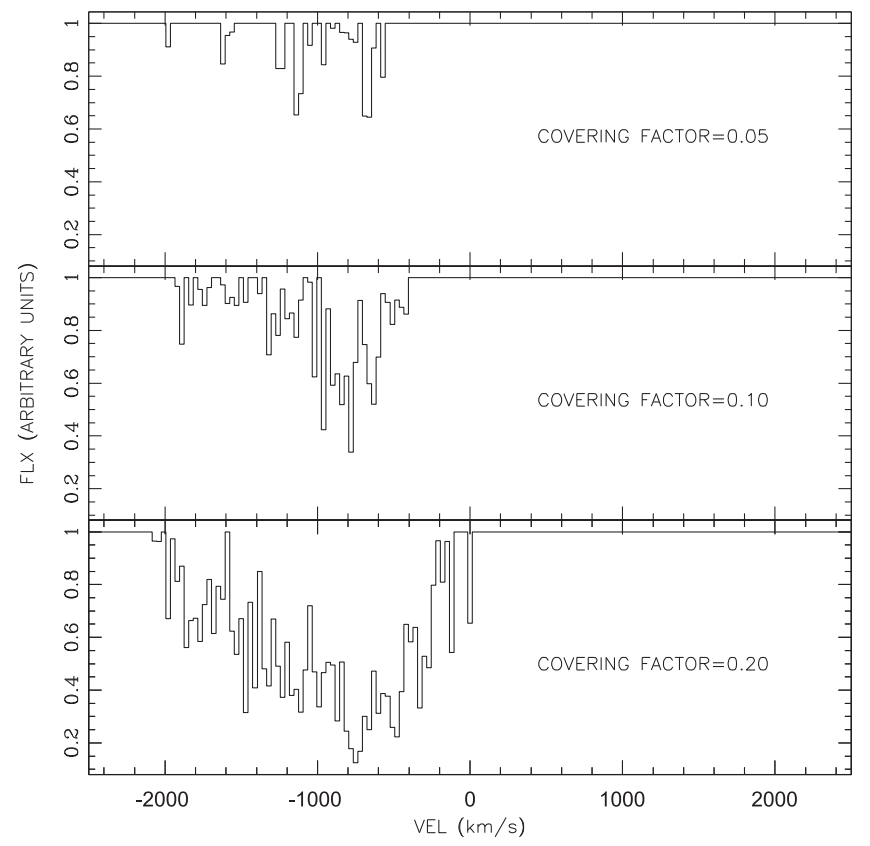

Figure 13. Simulation showing the increasing number of absorption structures for increasing covering factors (see text for more details).

studied in panchromatic high-resolution spectroscopy. The figure shows that structures are common to all of them at early decline as soon as the pseudo-photosphere has begun to recede and that similar structures are still visible at very late epochs once the ejecta is frozen in a high-ionization state. There is no one-to-one correspondence of the structures at the two epochs for the following: (1) the opacity of the ejecta is very different and at early phases is rapidly varying as the ejecta expand, and (2) the photosphere against which the absorptions are detected is different at the two epochs and is, in particular, much smaller (being no longer within the ejecta but matching instead the bloated WD photosphere) in the late nebular phase. Nonetheless, one can notice that the structure visible at $-1450 \mathrm{~km} \mathrm{~s}^{-1}$ in the T Pyx 37 day spectrum is the same as the 834 day spectrum. More generally, where we detect bands of absorbing structures at early epochs we detect absorbing structures later on. The largest velocities observed at the two epochs are comparable to those observed in the wings of the emission lines when their emission measure was sufficient. We are, therefore, always seeing the same unchanged distribution of clumps under different illumination, i.e., ionization degrees compatible with their local density and the light source underneath. Their absolute position in space is stretching linearly with time as the ejecta expand. This matches a Hubble flow, i.e., the structures are in ballistic expansion.

Another important feature of ballistic expansion is that the timescale is unique, independent of the radial distance within the ejecta. Since the recombination time depends on the electron density as $n_{e}^{-1}$, and the electron density varies as $n_{e} \sim r^{-3} \sim v_{\text {rad }}^{-3}$, at any epoch after the ejection there is a unique radial velocity (in the observer's frame) that corresponds to that density. It is an expected consequence that the dominant ionization stage of any species is confined at any time in a range of velocities that depends only on the maximum velocity of the ejecta and its mass. Hence, the radial velocity interval in which the discrete absorption features will be observed for any ion will always be about the same, among 


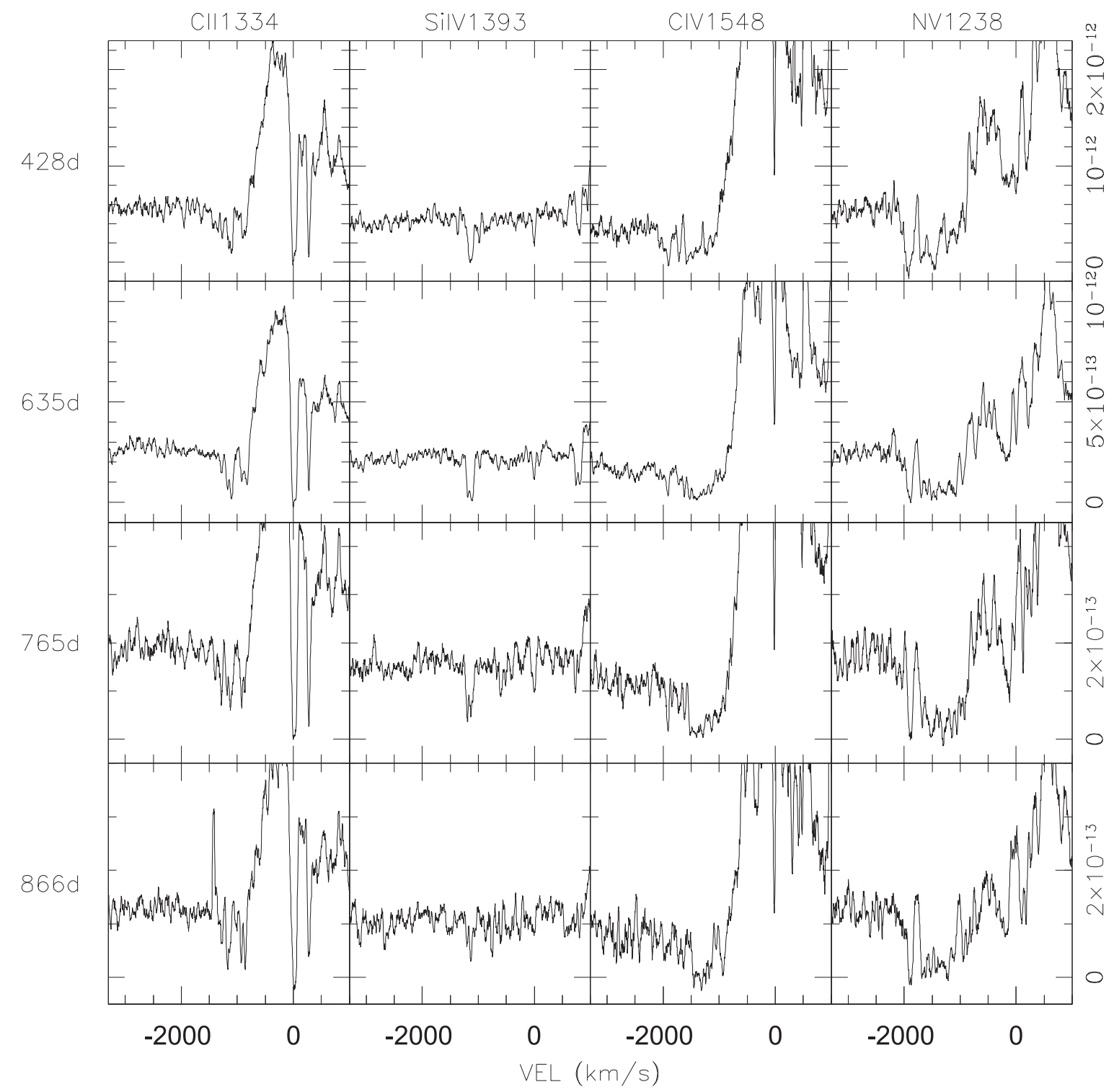

Figure 14. V339 Del ionization structures as evinced from the absorptions observed in the permitted resonant transitions. The transitions and the epoch of each observation are labeled on the figure side (top and left, respectively). On the right axis of the plot, fluxes are in $\operatorname{erg~cm}^{-2} \mathrm{~s}^{-1} \AA^{-1}$.

novae, even if the individual structures are different. For example, for $n_{e} \sim 10^{5} \mathrm{~cm}^{-3}$, as we have in the last epoch of V1369 Cen, the N V and C IV should be observed at around $v_{\mathrm{rad}} \approx-1500 \mathrm{~km} \mathrm{~s}^{-1}$.

\section{Discussion}

\subsection{Commonalities among Novae: The Model from the UV +Optical High-resolution Spectroscopic Monitoring}

The observational fact emerging from the analysis of the data sets presented in this and the previous works of the series is the presence of persistent absorption structures that do not move, in velocity space, with time. This is evident when comparing late nebular spectra of the same nova (e.g., Figure 30 in De Gennaro Aquino et al. 2014; Figures 12 and 14 in this paper). The persistence of the absorption structures is also shown by comparison of the spectra taken during early decline with those taken during late nebular stages (e.g., Figure 15), and by the evolution of the line profiles across time (e.g., Figure 16; Figures 2 and 4 in Shore et al. 2011; Figures 2 and 3 in Shore et al. 2016). The absorptions from low ionization energy ions visible in a given velocity interval at early stages are also present at later epochs in the resonance transitions of high ionization energy ions. They indicate clumps that have only changed their ionization state, while following the global expansion of the ejecta. The absorption troughs observed during the initial phases of the outburst break into complex absorption structures and tend to shift toward higher $v_{\text {rad. This }}$ matches a clumpy ejecta whose outer portions cool and recombine as the ejecta expand, and as the pseudo-photosphere recedes, the line of sight intercepts progressively fewer structures. Note that these absorptions can also display apparent inward motion or oscillations (e.g., McLaughlin 1957; Figure 1 in Williams et al. 2008; our Figure 16) depending on the nova characteristics and the cadence of the monitoring: each clump absorbs (at a given wavelength) when its density and the incident radiation from behind allow it.

Persistent structures that do not move in velocity space are, by definition, a Hubble/radial flow (or in ballistic expansion). That they are stationary at velocities well below the maximum 

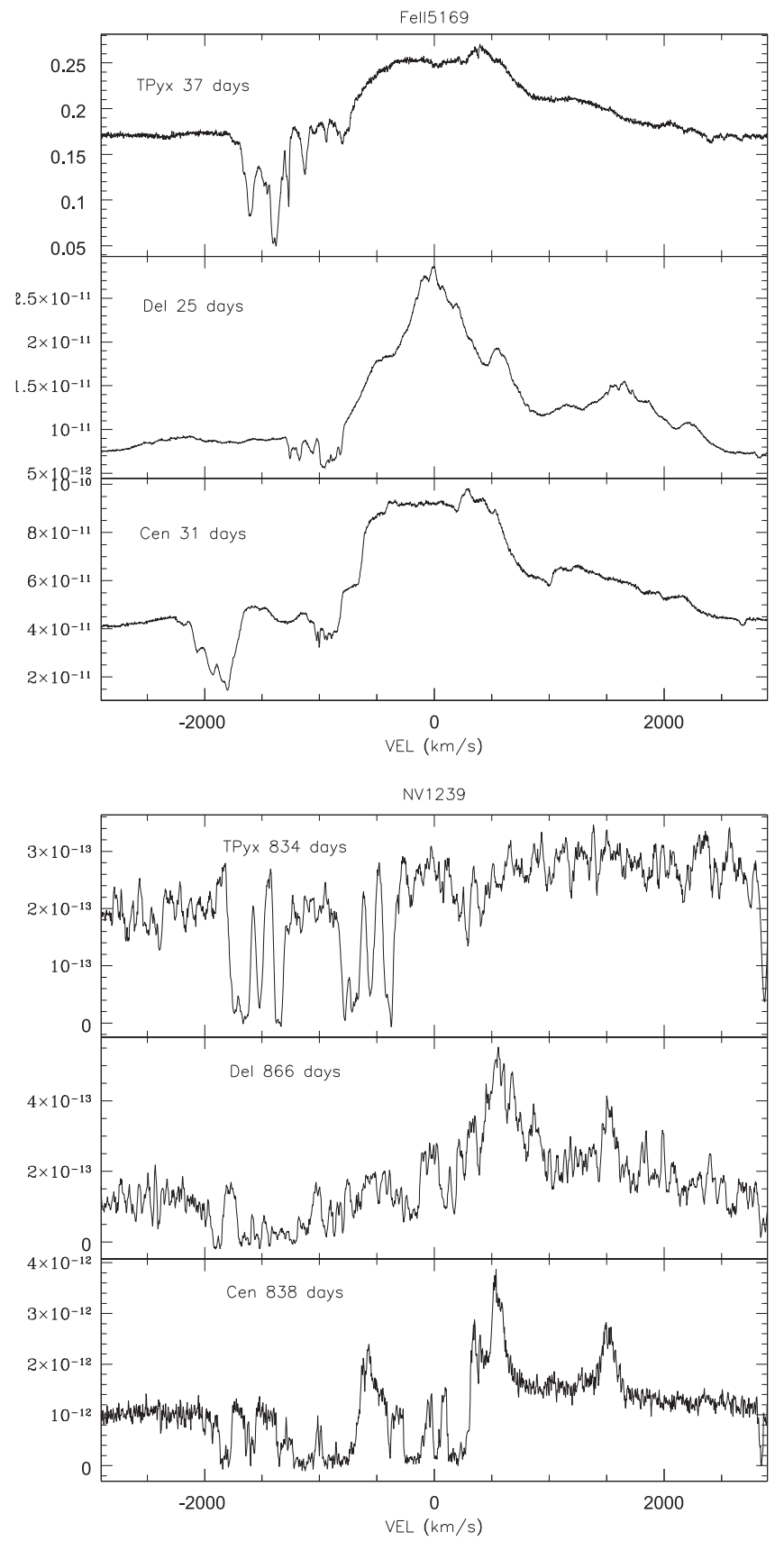

Figure 15. Absorption structures in the spectra of our "panchromatic novae" during early decline in the Fe II $\lambda 5169$ line (top) and during the late nebular phase in the $\mathrm{N}$ V $\lambda 1239$ transition (bottom).

velocity observed during early phases of the expansion is not compatible with a wind (e.g., Lamers \& Cassinelli 1999). Therefore, a major conclusion of our works is that, in the formation of the observable ejecta, there is no wind ejection at any phase and the ejecta are expanding undisturbed.

In the past, the absorption features observed at early stages in view of their apparent acceleration (despite some exceptions) were interpreted as subsequent shells ejected with increasing velocity. Were that the case, we would not be observing the same structures years after the outburst. It is interesting to note that Liimets et al. (2012) reach similar conclusions (ballistic expansion and little or no deceleration) analyzing the resolved clumps over the past $25 \mathrm{yr}$ of the $>100 \mathrm{yr}$ old nova GK Per.
Our monitoring shows that the measured line flux and the derived density in the late nebular spectra of our data sets match the evolution expected for a ballistic expansion. This same conclusion was previously reached by Shore et al. (1996) by analyzing the UV line flux of V1974 Cygni together with its $\mathrm{X}$-ray light curve.

An undisturbed ballistic expansion is incompatible with multiple ejections of shells or structures having increasing velocity (e.g., McLaughlin 1957; Friedjung 1987; Kimeswenger et al. 2008; Tanaka et al. 2011; Chomiuk et al. 2014; Arai et al. 2016). In addition, the velocity field of a ballistic expansion is incompatible with any geometry that implies acceleration or deceleration of the ejecta (e.g., an hourglass geometry).

It is known from imaging that nova ejecta are not spherical but elongated (e.g., Hjellming 1995; Slavin et al. 1995; Gill \& O'Brien 2000; Harman \& O'Brien 2003; Lane et al. 2007; Chesneau et al. 2011; Liimets et al. 2012; Chomiuk et al. 2014; Schaefer et al. 2014; Linford et al. 2015; Shara et al. 2015; Healy et al. 2017), yet the exact geometry is far from certain. The simplest geometry compatible with a nonspherical radial expansion is polar caps or a biconical form (the difference between the two depending on the thickness one considers). Combining this consideration with the clumpiness of the ejecta, we used a simple Monte Carlo code to mimic the observed line profiles. We adopt a biconical geometry with random clumps that obey a velocity and density distribution matching the ballistic expansion, and emissivity from recombination as appropriate for the late nebular spectra. Our series of papers has shown that such a geometry suffices in reproducing the gross line profiles we observe in high-resolution spectroscopy. Therefore, at this stage, there is no need to consider more complex geometries. The detailed reproduction of individual features would require real dynamical models, which are beyond the scope of this series.

An interesting test of the validity of our approach comes from the use of the adopted geometry in the computation of the ejecta mass and filling factor. They are computed adopting the identified emitting volume and integrating the $n \sim 1 / r^{3}$ density within such volume. Each epoch is treated independently, providing ejecta mass estimates that are the same within the uncertainties (e.g., Figure 14 of Shore et al. 2016).

The observed spectral evolution is a further validation of the proposed picture. At late phases the highest ions sample the outermost regions of the ejecta because the lower density there prevents recombination. Neutral or singly ionized transitions are favored in the densest region of the ejecta and are expected to be observable for several years in the inner regions of the most massive ejecta. Were the density following a different distribution, we would not have observed such an ionization structure.

\subsection{The Scenario at Other Wavelengths: A Qualitative Test}

Observations by the Fermi/LAT satellite have revealed that novae are capable of emitting $\gamma$-rays with energy $\geqslant 100 \mathrm{MeV}$ that are evidence of shocks among accelerated particles (Fermi Collaboration 2014; Cheung et al. 2016). Metzger et al. (2014) propose that the $100 \mathrm{MeV}$ emission arises from the collision of distinct ejections of gas having mass differences within an order of magnitude and relative velocity in the range $0.15-0.60$. These conditions match the observed range of velocities (from a few hundred to a few thousand kilometers per second; Figures 12, 14, and 15; see also Figures 29 and 30 of De Gennaro Aquino et al. 2014) and the measured local projected densities at any 


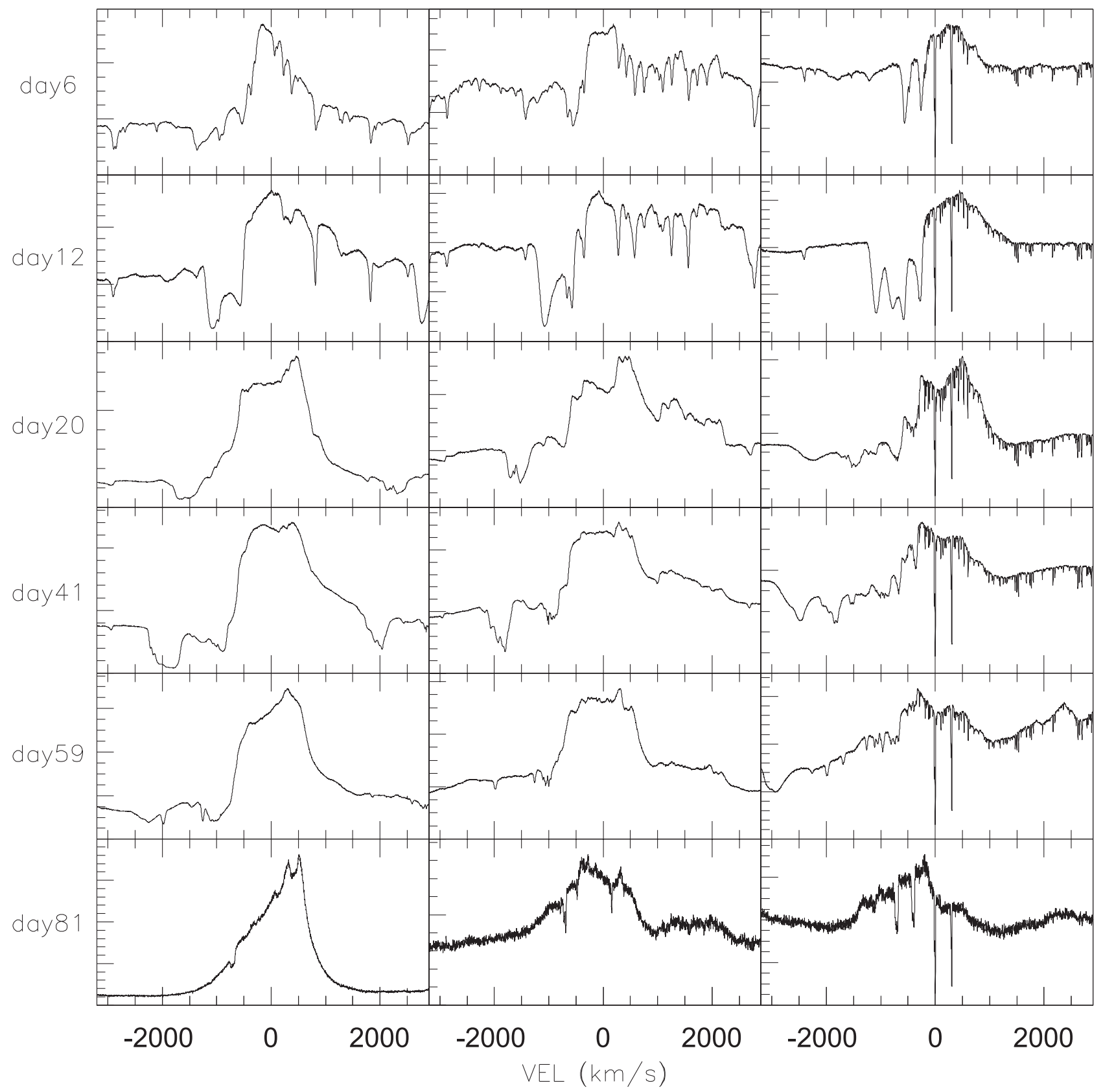

Figure 16. V1369 Cen early-decline line profiles.

given time (e.g., Figure 4). Hence, collisions between clumps during the ejection and the initial expansion could explain the $100 \mathrm{MeV}$ emission. More than that, intraclump collisions are expected in a single short-duration explosion that generates a stochastic range of clump velocities. The orientation and porosity of the ejecta and the density of individual clumps would then power the $\gamma$-ray and X-ray light curves. The modeling of those curves, like precise line profile fitting, would require assuming clump distributions, which are beyond the scope of this paper.

Centimeter-wavelength radio observations have shown morphological changes of the ejecta in resolved images (e.g., Hjellming 1996, and references therein; Chomiuk et al. 2014; Wendeln et al. 2017). Different morphologies observed in different epochs at different frequencies can be explained by differential evolution of the opacity in the spatially resolved nonspherical inhomogeneous sources, similarly to the changes in the line profiles during early stages of the outburst. Indeed, the "varying" radio images during the optically thick phase of the novae were explained by nonspherical single ejecta having a velocity gradient (e.g., Hjellming 1996, 1995). Global density gradients and inhomogeneous ejecta were instead inferred from radio observations deviating from the theoretical distribution expected for a uniform optically thick and isothermal gas (e.g., Seaquist \& Palimaka 1977). Morphological difference in resolved radio images of an optically thin gas (e.g., Chomiuk et al. 2014) maps the different emissivity of each region at a given frequency, similarly to the profile difference we observe in the optical in the late nebular phase (e.g., Figure 17, bottom panel; compare $\mathrm{NIV}$ ] to $\mathrm{H} \beta$ for an example of a dramatic difference). 


\section{Summary and Conclusion}

In this paper we have analyzed V1369 Cen nebular spectra and compared them with those of the novae observed with similar instrumentation in order to both determine the physical parameters of V1369 Cen ejecta and spot commonalities among novae that could allow the identification of a unified picture for their ejecta and ejecta kinematics.

Our quantitative results for the physical parameters of V1369 Cen are (1) $E(B-V)=0.15 \mathrm{mag}$, (2) distance in the range $1.8-2.4 \mathrm{kpc}$, (3) filling factor in the range $0.1-0.2$, (4) ejecta mass $\sim 1 \times 10^{-4} M_{\odot}$, and (5) anomalous $\mathrm{Ne}$ abundance and anomalously large $\mathrm{N} / \mathrm{C}$ abundance ratio.

Our effort toward a unified picture for nova ejecta is necessarily qualitative. It makes use of a single assumption (the single explosion event) and of the evidence that the ejecta are neither spherical nor homogeneous. The presence of stationary structures is, instead, new observational evidence from our data. Their interpretation requires that they constitute a ballistic expansion, contradicting any picture of wind-like ejecta. The observed kinematics automatically define a density distribution and, therefore, behaviors that can be verified (and/or predicted) through the monitoring. Examples are the outward propagation of a recombination wave during the early phase of the ejecta expansion (the iron curtain phase), the higher ionization degree being larger in the outskirts of the ejecta, the decline of the emission-line intensity once the SSS has turned off and the ejecta are dominated by recombination, and the frozen line profile and ionization structure, again, once the ejecta cool mainly by recombination. The biconical geometry globally constrains the ensemble of structures in the aspherical ejecta to reproduce the gross observable features (line width, maximum velocity, more or less "rectangular" or "saddle-shaped" emission line profiles). The resulting model, besides being able to explain the panchromatic high-resolution spectroscopic monitoring, is consistent with recent observations at other wavebands (e.g., $\gamma$-ray and radio). On the contrary, other proposed scenarios (e.g., colliding winds and shells), while capable of explaining those wavebands and/or individual observations or short-term monitoring, are incompatible with the data sets shown for V959 Mon, V339 Del, V1369 Cen, and even $\mathrm{T}$ Pyx in our series of papers.

We conclude by noting that while we have achieved a selfconsistent picture for the observable nova ejecta, the formation of the ejecta structures remains an open problem.

Based on observations made with (1) the NASA/ESA Hubble Space Telescope, obtained from the data archive at the Space Telescope Science Institute (STScI), which is operated by the Association of Universities for Research in Astronomy, Inc., under NASA contract NAS 5-26555; (2) the Nordic Optical Telescope, operated on the island of La Palma jointly by Denmark, Finland, Iceland, Norway, and Sweden, in the Spanish Observatorio del Roque de los Muchachos of the Instituto de Astrofisica de Canarias; (3) the ESO Very Large Telescope at the Paranal Observatory; and (4) the ESO/MPG $2.2 \mathrm{~m}$ in the La Silla Observatory.

The authors are grateful to Teddy Cheung, Jordi José JanUwe Ness, Julian Osborne, Francois Teyssier, and Brian Warner for having participated in the 2013 Pisa meeting, helping define this research program. E.M. warmly thanks Pierluigi Selvelli for the enlightening spectroscopic confrontations and the friendly "calories support." S.N.S. thanks Thomas
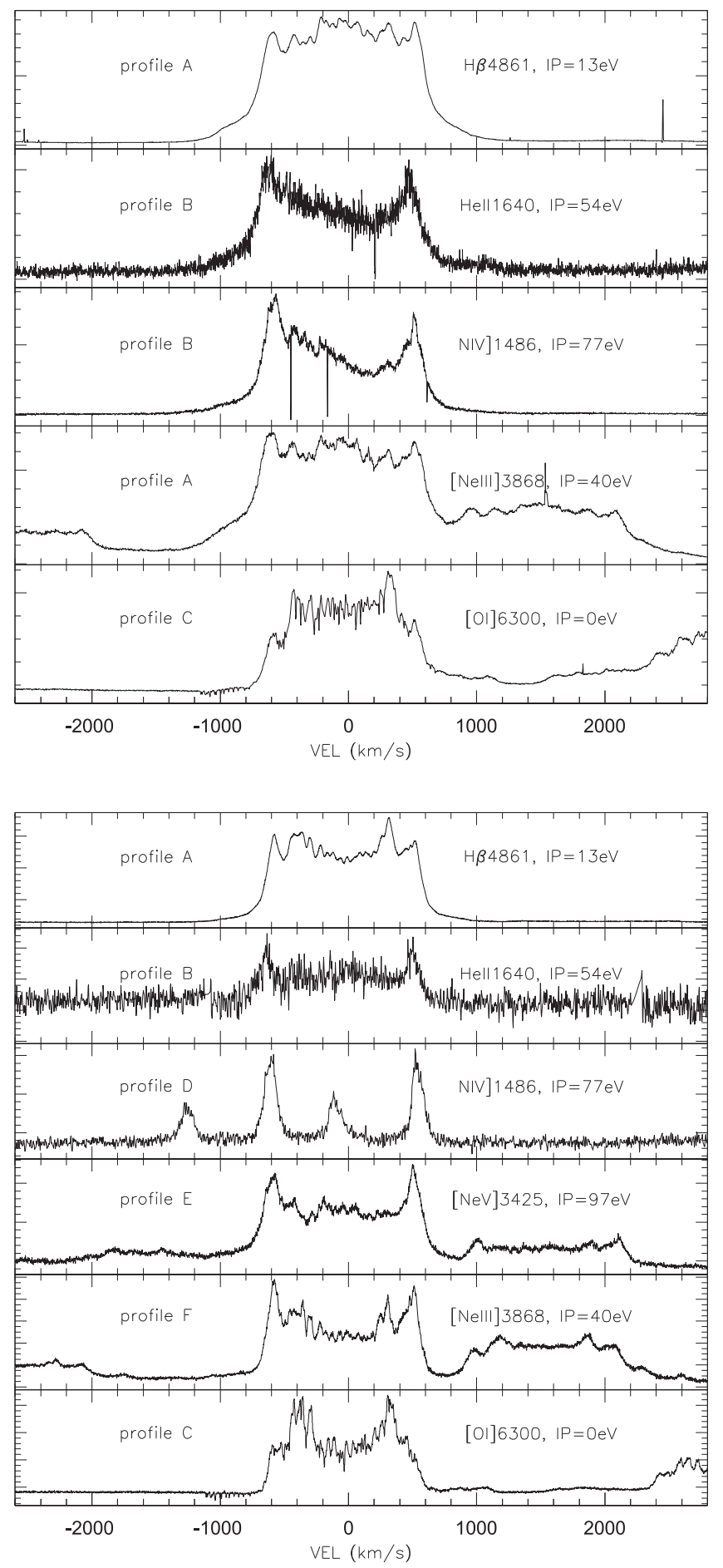

Figure 17. Master reference profiles used to aid the line identification in two illustrative epochs (top: day 252; bottom: day 836) of V1369 Cen spectral evolution.

Augusteijn, Bob Gherz, Pierre Jean, Paul Kuin, Sumner Starrfield, Fred Walter, and Laura Chomiuk for discussions; John Telting for his help with the NOT observations; and the Astronomical Institute of the Charles University for a visiting professorship. The authors thank the anonymous referee for the helpful critiques.

Facilities: HST(STIS), VLT(UVES), NOT(FIES), Swift (XRT), AAVSO.

Software: IRAF, Super Mongo, IDL. 


\section{Appendix Identified Lines and Their Profile Characteristics}

The use of high-resolution spectroscopy has revolutionized not only our understanding of the ejecta geometry, structure, and kinematics but also our ability to identify atomic species and their ions. It is possible to identify the species forming a blend by comparing peaks or structures and wings of the line profile. When there is still ambiguity on the transition identification, the profile characteristics, if discernible, can be used to solve the ambiguity since ions of similar ionization potential energy produce transitions of similar profile. In addition, the ability of distinguishing different profile types allows us to recognize when a transition is "replaced" by another owing to the change of ionization state of the ejecta. In Figure 17 we present a sample of "master" lines for the first (day 252 , top panel) and the last (day 836, bottom panel) epoch. Those profiles were used to help the line identification listed in Tables 4, 5, and 6; the observed lines are listed together with their profile types. Note that the profile types at the two epochs are independent because the emission measure of a given transition changes with time, affecting the profile. Hence, each set of profile types refers exclusively to the epoch in which it is used.

Table 4

Observed Transitions and Their Line Profile Typology (Cross-check with Profiles of the Corresponding Epoch in Figure 17) for the UV Range of the Spectrum

\begin{tabular}{lccc}
\hline \hline$\lambda(\AA)$ & Transition & Day 252 Profile & Day 838 Profile \\
\hline 1239,1243 & N V & B & D \\
1302,1305 & O I & w & $\ldots$ \\
1323 & $?$ & A & $\ldots$ \\
1336,1334 & C II & A: & D \\
1343 & O III & A & $\ldots$ \\
1355 & O I: & A & $\ldots$ \\
1371 & O V & B & A: \\
1389 & $?$ & A & $\ldots$ \\
1401,1405 & O IV & B & D \\
1411 & $?$ & A/B & $\ldots$ \\
1486 & N IV & B & D \\
1548,1551 & C IV & B & D \\
1526,1529 & $?$ & A: & $\ldots$ \\
1575 & [Ne V] & C: & $\ldots$ \\
1601 & [Ne IV] & B & $\ldots$ \\
1619 & C III & B & $\ldots$ \\
1640 & He II & B & B \\
1661,1666 & O III & B & W \\
1718 & N IV & B & $\ldots$ \\
1749,1751 & N III & B: & $\ldots$ \\
1884,1892 & Si III & W & $\ldots$ \\
1907,1909 & C III & B & D \\
2139,2143 & N II & A: & $\ldots$ \\
2188 & $?$ & A: & $\ldots$ \\
2297 & C III & B & W \\
2327,2328 & C II & A: & $\ldots$ \\
2344 & $?$ & B: & $\ldots$ \\
2470 & O II & A & wD \\
2647 & N IV & B & $\ldots$ \\
2735 & $?$ & B & $\ldots$ \\
2784 & $?$ & B & wD: \\
2796,2803 & Mg II & A: & $\ldots$ \\
2827 & $?$ & $?$ & $\ldots$ \\
2837 & C II: & B & $\ldots$ \\
3049 & $?$ & B & $\ldots$ \\
\hline & & & \\
\hline & & &
\end{tabular}

Note. The ":" notation is applied to those measurements or identification or profile assignment that are uncertain. The "b" assignment means that there is a blend, while the "w" indicates weak lines.
Table 5

Same as Table 4, but for the Visible Band

\begin{tabular}{|c|c|c|c|}
\hline \multirow[b]{2}{*}{$\lambda(\AA)$} & \multicolumn{3}{|c|}{ Day 252} \\
\hline & Transition & Profile & Day 838 Profile \\
\hline 3760 & O III & B & $\mathrm{bB} / \mathrm{E}$ \\
\hline 3771 & $\mathrm{H}_{11}$ & $\mathrm{~b}$ & $\mathrm{bA}$ \\
\hline 3798 & $\mathrm{H}_{10}$ & A & $\mathrm{bA}$ \\
\hline 3835 & $\mathrm{H} \eta$ & A & A \\
\hline 3869 & {$[\mathrm{Ne}$ III] } & A & $\mathrm{F}$ \\
\hline 3889 & $\mathrm{H} \zeta$ & A & A \\
\hline 3893, 3896 & {$[\mathrm{Fe} \mathrm{V}]$} & $\ldots$ & $\mathrm{b}$ \\
\hline $3968+3970$ & {$[\mathrm{Ne} \mathrm{III}]+\mathrm{H} \varepsilon$} & $\mathrm{b}$ & $\mathrm{b}$ \\
\hline 3995 + 4004: & $\mathrm{N}$ II+N III & A & $\mathrm{b}$ \\
\hline 4026 & He I & $\mathrm{bA}$ & {$[\mathrm{Fe} \mathrm{v}] 4027, \mathrm{~b}$} \\
\hline 4041 & $\mathrm{~N}$ II & A & $\mathrm{b}$ \\
\hline 4072,4076 & O II & $\mathrm{b}$ & {$[\mathrm{Fe} \mathrm{V}] 4072, \mathrm{~b}$} \\
\hline $4102+4097,4104$ & $\mathrm{H} \gamma+\mathrm{N}$ III & $\mathrm{b}$ & $\mathrm{b}$ \\
\hline $4144+4152$ & $\mathrm{He} \mathrm{I}+\mathrm{C}$ III & w & w \\
\hline 4181: & $?$ & $\mathrm{~b}$ & $\mathrm{~b}$ \\
\hline$+4190+4196,4200$ & $+\mathrm{O}$ II $+\mathrm{N}$ III & & \\
\hline 4237,4242 & N II & $\mathrm{b}$ & $\mathrm{b}$ \\
\hline 4267, 4274:+4287: & $\mathrm{C} \mathrm{II}+?$ & bA: & bA \\
\hline 4320 & $\mathrm{O}$ II & $\mathrm{b}$ & $\mathrm{w}$ \\
\hline 4341 & $\mathrm{H} \gamma$ & A & A \\
\hline 4363 & [O III] & A & $\mathrm{F}$ \\
\hline 4379 & $\mathrm{~N}$ III & A: & $\mathrm{B}$ \\
\hline 4415,4417 & O II: & $\mathrm{b}$ & $\mathrm{w}$ \\
\hline $4447+4448$ & $\mathrm{~N}$ II+O II & $\mathrm{b}$ & \\
\hline 4472 & He I & A & $\mathrm{A}$ \\
\hline $\begin{array}{c}4511 / 15 / 24 / 31 / \\
35+4542\end{array}$ & $\mathrm{~N}$ III+He II & $\mathrm{b}$ & $\mathrm{b}$ \\
\hline 4591 & O II & $\mathrm{b}$ : & $\mathrm{b}$ \\
\hline $4603+4606$ & N v:+? & $\mathrm{b}$ & 4607?, bA: \\
\hline $4634.2,4640.6+4649$ & $\mathrm{~N}$ III+O II & $\mathrm{b}$ & bB \\
\hline 4658 & [Fe III]: & $\mathrm{b}$ & $\mathrm{b}$ \\
\hline 4686 & He II & $\mathrm{A} / \mathrm{B}$ & $\mathrm{B}$ \\
\hline 4702:+4706: & $\mathrm{O} \mathrm{II}+?$ & $\mathrm{~b}$ & $+4699, \mathrm{~b}$ \\
\hline 4714,4725 & [Ne IV] & $\mathrm{b}$ & $\ldots$ \\
\hline 4740 & [Ar IV] & $\ldots$ & $\mathrm{B}$ \\
\hline 4803 & C II & $\mathrm{w}$ & $\mathrm{A} / \mathrm{C}$ \\
\hline 4861 & $\mathrm{H} \beta$ & A & A \\
\hline 4894 & {$[\mathrm{Fe} \mathrm{VII}]$} & $\ldots$ & wB: \\
\hline 4959 & {$[\mathrm{O} \mathrm{III}]$} & $\mathrm{A}$ & $\mathrm{F}$ \\
\hline 5007 & [O III] & A & $\mathrm{F}$ \\
\hline 5041,5056 & Si II: & $\mathrm{b}$ & bB: \\
\hline $5147+5158$ & $\begin{array}{c}{[\mathrm{Fe} \mathrm{VI}]} \\
+[\mathrm{Fe} \mathrm{VII}]\end{array}$ & $\mathrm{b}$ & {$[\mathrm{Fe} \mathrm{VI}], \mathrm{bB}:$} \\
\hline 5176 & {$[\mathrm{Fe} \mathrm{VI}]$} & $\ldots$ & $w B$ \\
\hline 5309 & {$[\mathrm{Ca} \mathrm{V}]$} & $\mathrm{B}$ & $\mathrm{E}$ \\
\hline 5336 & {$[\mathrm{Fe} \mathrm{VI}]$} & $\ldots$ & $\mathrm{E}:$ \\
\hline 5412 & He II & A & $+[\mathrm{Fe} \mathrm{VI}] 5425, \mathrm{~b}$ \\
\hline 5486 & {$[\mathrm{Fe} \mathrm{VI}]$} & $\ldots$ & wB \\
\hline $5530+5534$ & $\mathrm{~N}$ II $+[\operatorname{Ar} \mathrm{X}]$ & $\mathrm{b}$ & w \\
\hline 55945615 & $?$ & $\mathrm{~B}$ & O III5592, A/B \\
\hline 5632 & {$[\mathrm{Fe} \mathrm{VI}]$} & & $w B / E$ \\
\hline $5668,5676,5679.6$ & $\mathrm{~N}$ II & $\mathrm{b}$ & $\mathrm{b}+[\mathrm{Fe} \mathrm{VI}]:$ \\
\hline 5721 & {$[\mathrm{Fe} \mathrm{VII}]$} & $\mathrm{B}$ & $\mathrm{B} / \mathrm{E}$ \\
\hline 5755 & {$[\mathrm{~N}$ II $]$} & $\mathrm{C}$ & $\ldots$ \\
\hline 5801,5812 & C IV & $\mathrm{b}$ & $\ldots$ \\
\hline 5876 & $\mathrm{He} \mathrm{I}$ & A & A \\
\hline 5932,5942 & $\mathrm{~N}$ II & A & $\mathrm{w}$ \\
\hline 6086 & {$[\mathrm{Ca} \mathrm{V}]$} & $\mathrm{B}$ & $\mathrm{E}$ \\
\hline 6300 & [O I] & $\mathrm{C}$ & $\mathrm{C}$ \\
\hline $6364+6346,6371$ & {$[\mathrm{O} \mathrm{I}]+\mathrm{Si} \mathrm{II}$} & $\mathrm{b}$ & {$[\mathrm{O} \mathrm{I}], \mathrm{C}$} \\
\hline $6482+6460:+6501$ & $\mathrm{~N} \mathrm{II+?}$ & $\mathrm{bA}$ & w \\
\hline 6563 & $\mathrm{H} \alpha+[\mathrm{N} \mathrm{II}]$ & $\mathrm{bA}$ & {$[\mathrm{N}$ II $], \mathrm{A} / \mathrm{C}$} \\
\hline 6675 & He I & A & A \\
\hline
\end{tabular}


Table 5

(Continued)

\begin{tabular}{|c|c|c|c|}
\hline \multirow[b]{2}{*}{$\lambda(\AA)$} & \multicolumn{3}{|c|}{ Day 252} \\
\hline & Transition & Profile & Day 838 Profile \\
\hline 6747 & ? & $\mathrm{A} / \mathrm{B}:$ & $\mathrm{w}$ \\
\hline 7006 & [Ar V] & $\mathrm{A} / \mathrm{B}$ & $\mathrm{B} / \mathrm{E}$ \\
\hline 7063 & $\mathrm{He} \mathrm{I}$ & A & A \\
\hline $7136+7150 / 2$ & $\begin{array}{l}\text { [Ar III] } \\
\text { +O II: }\end{array}$ & $\mathrm{b}$ & {$[\mathrm{Ar}] \mathrm{F}$} \\
\hline $7232+7236$ & $\begin{array}{c}\text { C II, } \\
{[\text { Ar IV] too? }}\end{array}$ & A & [Ar IV], A \\
\hline 7320,7331 & [O II] & $\mathrm{b}$ & $\mathrm{b}$ \\
\hline $7705+7717+7726$ & $\begin{array}{c}\text { N II]: } \\
\text { or N III]: }\end{array}$ & $\mathrm{b}$ & $\begin{array}{c}{[\text { Ar III]: }} \\
7704+7714 ? \\
+7751 ?\end{array}$ \\
\hline 7774 & O I & b & $\ldots$ \\
\hline $8237+8196:$ & $\mathrm{He}$ II $+\mathrm{C}$ III? & $\mathrm{b}$ & He II, A: \\
\hline 8446 & O I & $\mathrm{C}$ & w \\
\hline 8545 & $\mathrm{~Pa}_{14}$ & A & $\mathrm{a}$ \\
\hline 8598 & $\mathrm{~Pa}_{13}$ & A & a \\
\hline $8665+8680 / 3:$ & $\mathrm{Pa}_{14}+\mathrm{N} \mathrm{I}:$ & $\mathrm{b}$ & b \\
\hline 8750 & $\mathrm{~Pa}_{12}$ & A & bA \\
\hline 8861 & $\mathrm{~Pa}_{11}$ & c & A \\
\hline 8926 & $?$ & A & $\ldots$ \\
\hline 9015 & $\mathrm{~Pa}_{10}$ & A & A \\
\hline $9068 / 70$ & [S III]: & bA & [S III], A \\
\hline
\end{tabular}

Notes.

a In the gap of the UVES red CCD mosaic.

b Partially in the UVES red CCD mosaic.

${ }^{c}$ Partially in the gap between the FEROS orders.

Table 6

Continuation of Table 5 for the Wavelength Range Covered by UVES (Day 838 Observation)

\begin{tabular}{|c|c|c|}
\hline$\overline{\lambda(\AA)}$ & Transition & $\overline{\text { Day } 838 \text { Profile }}$ \\
\hline 3133 & O III & $\mathrm{F} / \mathrm{B}$ \\
\hline 32033 & He II & B \\
\hline 3266,32616 & O III & bB \\
\hline 3312, 3299 & O III & bB \\
\hline $3341+3346$ & $\mathrm{O} \mathrm{III}+[\mathrm{Ne} \mathrm{V}]$ & bB \\
\hline 3381,3386 & O IV & $\mathrm{bB}$ \\
\hline $3404,3412+3426$ & $\mathrm{O} \mathrm{IV}+[\mathrm{Ne} \mathrm{V}]$ & $\mathrm{bE}$ \\
\hline 3444 & $\mathrm{O}$ III & $\mathrm{B}$ \\
\hline 3479: & N IV & w \\
\hline 3587: & {$[\mathrm{Fe} \mathrm{VII}]$} & $\mathrm{w}$ \\
\hline 3703 & O III & $w b$ \\
\hline $3726+3729$ & $\mathrm{O} \mathrm{IV}+[\mathrm{O} \mathrm{II}]$ & $\mathrm{b}$ \\
\hline $3750+3755$ & $\mathrm{H}_{12}+\mathrm{O}$ III & $\mathrm{b}$ \\
\hline 9229.0 & $\mathrm{~Pa}_{9}$ & $\mathrm{~A}$ \\
\hline 9532.1 & [S III] & A \\
\hline 9903.5 & $?$ & $\mathrm{w}$ \\
\hline 10049.6 & $\mathrm{~Pa}_{8}$ & A \\
\hline 10123.6 & He II & B \\
\hline 10395.6, 10404.1 & {$[\mathrm{~N} \mathrm{I}]$} & $\mathrm{w}$ \\
\hline
\end{tabular}

\section{ORCID iDs}

Elena Mason (ㄷ) https://orcid.org/0000-0003-3877-0484

Kim Page (i) https://orcid.org/0000-0001-5624-2613

Greg J. Schwarz (1) https://orcid.org/0000-0002-0786-7307

\section{References}

Arai, A., Kawakita, H., Shinnaka, Y., et al. 2016, AJ, 830, 30

Asplund, M., Grevesse, N., Sauval, A. J., et al. 2009, ARA\&A, 47, 481

Banerjee, D. P. K., Srivastava, M. K., Ashok, N. M., et al. 2016, MNRAS, 455,109

Cardelli, J. A., Clayton, G. C., \& Mathis, J. S. 1989, ApJ, 345, 245

Casanova, J., José, J., García-Berro, E., \& Shore, S. N. 2016, A\&A, 595, 28

Cassatella, A., Gonzales-Riestra, R., \& Selvelli, P. 2004a, "Classical Novae," INES Access Guide N.3 (Noordwijk: ESA)

Cassatella, A., Lamers, H. J. G. L. M., Rossi, C., et al. 2004b, A\&A, 420, 571

Chesneau, O., Meilland, A., Banerjee, D. P. K., et al. 2011, A\&A, 534, 11

Cheung, C. C., Jean, P., Shore, S. N., et al. 2016, ApJ, 826, 142

Chochol, D., Grygar, J., Pribulla, T., et al. 1997, A\&A, 318, 908

Chomiuk, L., Linford, J. D., Yang, J., et al. 2014, Natur, 514, 339

De Gennaro Aquino, I., Shore, S. N., Schwarz, G. J., et al. 2014, A\&A, 562, A28

Downen, L. N., Iliadis, C., José, J., \& Starrfield, S. 2013, ApJ, 762, 105

Eyres, S. P. S., Heywood, I., O’Brien, T. J., et al. 2005, MNRAS, 358, 1019

Fermi Collaboration 2014, Sci, 345, 554

Friedjung, M. 1966a, MNRAS, 131, 447

Friedjung, M. 1966b, MNRAS, 132, 143

Friedjung, M. 1987, A\&A, 180, 155

Gill, C. D., \& O'Brien, T. J. 1999, MNRAS, 307, 677

Gill, C. D., \& O'Brien, T. J. 2000, MNRAS, 314, 175

Harman, D. J., \& O'Brien, T. J. 2003, MNRAS, 344, 1219

Harvey, E., Redman, M. P., Boumis, P., et al. 2016, A\&A, 595, 64

Healy, F., O'Brien, T. J., Beswick, R., et al. 2017, MNRAS, 469, 3976

Hjellming, R. M. 1990, Proc. Coll. 122 of the IAU 369, Physics of Classical Novae, ed. A. Cassatella \& R. Viotti (Berlin: Springer-Verlag), 169

Hjellming, R. M. 1995, in Proc. Conf. 205, Cataclysmic Variables, ed. A. Bianchini, M. della Valle, \& M. Orio (Dordrecht: Kluwer), 139

Hjellming, R. M. 1996, in ASP Conf. 93, Radio emission from the stars and the sun, ed. A. R. Taylor \& J. M. Paredes (San Francisco, CA: ASP), 174

Hutchings, J. B. 1970, PDAO, 13, 397

José, J., \& Shore, S. N. 2008, Classical Novae (2nd ed.; Cambridge: Cambridge Univ. Press)

Kafka, S. 2017, Observations from the AAVSO International Database, https://www.aavso.org

Kalberla, P. M. W., Burton, W. B., Hartmann, D., et al. 2005, A\&A, 440, 775

Kalberla, P. M. W., \& Haud, U. 2015, A\&A, 578, A78

Kimeswenger, S., Dalnodar, S., Knapp, A., et al. 2008, A\&A, 479, 51

Lamers, H. J. G. L. M., \& Cassinelli, J. P. 1999, Introduction to Stellar Winds (Cambridge: Cambridge Univ. Press)

Lane, B. F., Retter, A., Eisner, J. A., et al. 2007, ApJ, 699, 1150

Liimets, T., Corradi, R. L. M., Santander-García, M., et al. 2012, ApJ, 761, 34

Linford, J. D., Ribeiro, V. A. R. M., \& Chomiuk, L. 2015, ApJ, 805, 136

Livio, M., \& Truran, J. W. 1994, ApJ, 425, 797

McLaughlin, D. B. 1957, VA, 2, 1147

McLaughlin, D. B. 1964, AnAp, 27, 450

Metzger, B. D., Hascoët, R., Vurm, I., et al. 2014, MNRAS, 442, 713

Munari, U., Ribeiro, V. A. R. M., Bode, M. F., et al. 2011, MNRAS, 410, 525

Munari, U., \& Zwitter, T. 1997, A\&A, 318, 269

Nussbaumer, H., \& Schild, H. 1979, A\&A, 75, L17

Nussbaumer, H., \& Schild, H. 1981, A\&A, 101, 118

O’Brien, T. J., \& Bode, M. F. 2008, Classical Novae (2nd ed.; Cambridge: Cambridge Univ. Press)

Page, K. L., Osborne, J. P., Wagner, R. M., et al. 2013, ApJL, 768, L26

Payne-Gaposchkin, C. 1957, The Galactic Novae (Amsterdam: North-Holland) Payne-Gaposchkin, C., \& Menzel, D. H. 1938, Har. Circ., 428, 1

Ribeiro, V. A. R. M., Darnley, M. J., Bode, M. F., et al. 2011, MNRAS, 412, 1701

Ribeiro, V. A. R. M., Munari, U., \& Valisa, P. 2013, ApJ, 768, 49

Schaefer, G. H., Brummelaar, T. T., Gies, D. R., et al. 2014, Natur, 515, 234

Schwarz, G. J. 2014, in ASP Conf. 490, Stella Novae: Past and Future Decades, ed. P. A. Woudt \& W. A. R. M. Ribeiro (San Francisco, CA: ASP), 101

Scott, A. D., Duerbeck, H. W., Evans, A., et al. 1995, A\&A, 296, 439

Seaquist, E. R., \& Palimaka, J. 1977, ApJ, 217, 781

Shara, Michael M., Zurek, D., Schaefer, B. E., et al. 2015, ApJ, 805, 148

Shore, S. N. 2013, A\&A, 559, L7

Shore, S. N., Augusteijn, T., Ederoclite, A., et al. 2011, A\&A, 533, L8

Shore, S. N., De Gennaro Aquino, I., Schwarz, G. J., et al. 2013a A\&A, 553, 123

Shore, S. N., Mason, E., Schwarz, G. J., et al. 2016, A\&A, 590, 123 
Shore, S. N., Schwarz, G. J., De Gennaro Aquino, I., et al. 2013b A\&A, 549,140

Shore, S. N., Starrfield, S., \& Sonneborn, G. 1996, ApJL, 463, L21

Slavin, A. J., O'Brien, T. J., \& Dunlop, J. S. 1995, MNRAS, 276, 353

Starrfield, S., Iliadis, C., \& Hix, W. R. 2016, PASP, 128, 1001

Tanaka, J., Nogami, D., Fujii, M., et al. 2011, PASJ, 63, 159
Vanlandingham, K. M., Schwarz, G. J., Shore, S. N., et al. 2001, AJ, 121,1126

Wendeln, C., Chomiuk, L., Finzell, T., et al. 2017, ApJ, 840, 110

Williams, P. M., Longmore, A. J., \& Geballe, T. R. 1996, MNRAS, 279, 804

Williams, R. E. 1992, AJ, 104, 725

Williams, R. E., Mason, E., Della Valle, M., et al. 2008, ApJ, 685, 451 